\title{
Indicadores por y para el desarrollo sostenible, un estudio de caso
}

\section{Indicators by and for sustainable development, a case study}

\author{
José A. Sotelo*, Alfredo Tolón** y Xavier Lastra**
}

\section{PRIMERA APROXIMACIÓN}

Los indicadores y su metodología están condicionados en cada momento por los conceptos, que a su vez dependen de las ideas y de los intereses implícitos en cada visión y misión, y en última instancia en cada concepción del mundo. Hemos de basarnos por tanto en la evolución del consenso de intereses e ideas que subyace en dicha concepción del mundo y en los respectivos conceptos para llegar a trazar una tipología de los indicadores del desarrollo sostenible desde su puesta en la agenda mundial por parte de la Comisión Brundtland. Evolución que nos llevaría a añadir a la emergencia de lo que Tamames llamó conciencia medioambiental, otras tres dimensiones superpuestas posteriormente: la económica, la social y la institucional (Matias, 2004).

Aun así, muchos de los aspectos de las conceptualizaciones, e incluso de los objetivos de acción declarados por las políticas inicialmente medioambientales y que ahora tienden a desembocar en políticas de desarrollo sostenible, no son todavía cuantificables, y en consecuencia no pueden aspirar siquiera a ser com-

\footnotetext{
* Instituto Universitario de Ciencias Ambientales, Universidad Complutense de Madrid. España (jasotelo@ghis.ucm.es).

** Departamento de Ingeniería Rural, Área de Proyectos de Ingeniería Universidad de Almería. España (atolon@ual.es y xlastra@ual.es).

Proyecto CICYT: CGL2007-63779
} 
parables internacionalmente. La conciencia de la insostenibilidad del sistema dominante ante el cambio ambiental global nos impele a la búsqueda de respuestas estratégicas, mediante un estilo redesarrollo que sea verdaderamente alternativo frente al modelo insostenible vigente. Desde esta perspectiva, los indicadores medioambientales y económicos, están hoy por hoy más logrados y son más fácil objeto de consenso en la confrontación de intereses, que los relativos a las perspectivas social e institucional, las últimas en ser incorporadas al concepto de sostenibilidad y a sus objetivos de acción (Matías, 2004).

No debemos olvidarnos que la sostenibilidad es equiparable al concepto ecológico de «resilencia» (capacidad que tienen los sistemas para adaptarse al cambio, para mantener su integridad, vencer los colapsos o las fluctuaciones externas y recuperarse en el tiempo). Es este contexto en el que el proceso de generación y aplicación de los sistemas de indicadores, en el marco estratégico de la sostenibilidad, se nos presentan como una herramienta de notable utilidad; desde una perspectiva diacrónica pueden identificarse sistemas de primera, segunda, y tercera generación. Los primeros, desarrollaban y comunicaban indicadores ambientales, incluyendo la aproximación por medios (aire, agua, tierra y biodiversidad), por objetivos (acordes con mandatos legales y administrativos, Agenda 21) y por sectores (transporte, turismo, industria, etc...). Posteriormente en los sistemas de segunda y, actualmente, en los de tercera generación, el desarrollo y la comunicación se basan en la creación de nuevos índices que sintetizan de forma práctica la información del desarrollo sostenible (Gallopín, 2006).

\section{a) Sistemas de primera generación}

Se originan en la década de los ochenta, del pasado siglo, a partir de las publicaciones recogidas por la OCDE, y se caracterizan por ser muy teóricos y exclusivamente ambientales. Los principales «marcos ordenadores» utilizados fueron:

- Presión-Estado-Respuesta (PER)

- Fuerza Motriz-Estado-Respuesta (FER)

- Fuerza Motriz-Presión-Estado-Respuesta (FPER)

- Fuerza Motriz-Presión-Estado-Impacto-Respuesta (FPEIR)

De ellos, los más utilizados fueron el PER, por la OCDE, y el FPEIR, por la Agencia Europea de Medio Ambiente (AEMA).

El Sistema de Indicadores de la OCDE, y su marco ordenador PER (figura 1) está compuesto por indicadores considerados parciales, que dan cuenta del fe- 
nómeno complejo desde un sector productivo o bien desde la singularidad o un número reducido de dimensiones. Su importancia radica en la obtención de indicadores con un nivel de rigor y calidad similar a los indicadores económicos y sociales, desarrollados anteriormente, y su aplicación se limita a nivel internacional, de especial manera en países desarrollados. Es uno de los pioneros en el desarrollo de indicadores a nivel mundial, y su trabajo aporta una visión interesante que vincula los problemas y oportunidades ambientales a los procesos económicos (Quiroga, 2001).

El Sistema de Indicadores de la Agencia Europea de Medio Ambiente (AEMA), fue creado en 2004, con la finalidad de establecer un sistema manejable y estable de elaboración de informes basados en indicadores, dar prioridad a las mejoras de calidad y cobertura geográfica de los flujos de datos, especialmente los flujos de datos prioritarios de la Red de Observación e Información Medioambiental de Europa (EIONTE), y racionalizar las aportaciones de la AEMA y EIONET a otras iniciativas de indicadores europeos y mundiales (MMA, 2006). Utiliza el marco ordenador FPEIR (figura 2) para

FIGURA 1

MODELO PRESIÓN - ESTADO - RESPUESTA

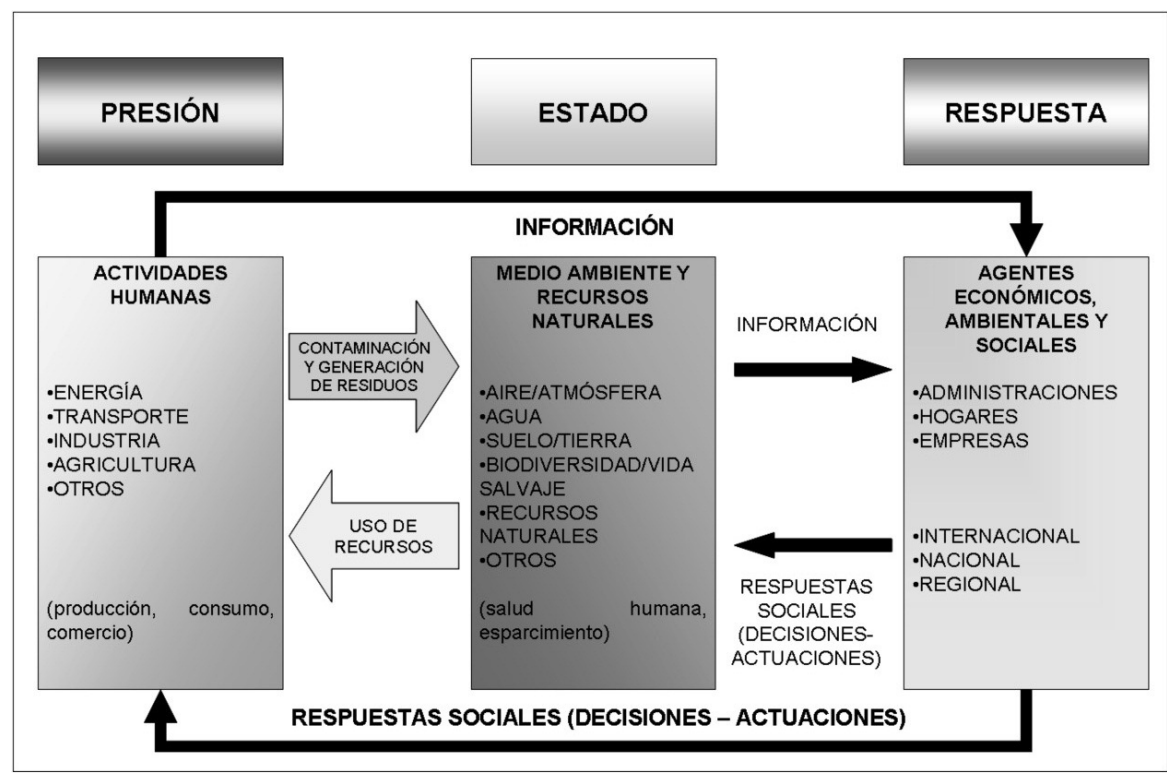

Fuente: Elaboración propia a partir de: MMA, 2000; OECD, 2003. 
sentar las bases del análisis de los factores interrelacionados que afectan al medio ambiente. Contempla indicadores básicos que abarcan seis temas medioambientales (contaminación atmosférica y agotamiento del ozono, cambio climático, residuos, agua, biodiversidad y medio terrestre) y cuatro sectores (agricultura, energía, transporte y pesca) sin desarrollar indicadores sociales ni de salud. Los informes anuales se publican a través de los informes «Environmental Signals», y se caracterizan por utilizar un leguaje no técnico, para abarcar una mayor audiencia. La AEMA no realiza investigaciones medioambientales por sí misma, sino que trabaja a partir de datos proporcionados por los países. Parte de la información llega a través de organizaciones como Eurostat (la Oficina de Estadística de la Comisión) o la Organización Mundial de la Salud (OMS). El resto de la información se recopila en gran parte a través de EIONET, un enorme recurso adicional de cuya creación y coordinación es responsable la AEMA. El ámbito geográfico de aplicación del Sistema de Indicadores de la Agencia Europea de Medio Ambiente es el nacional.

FIGURA 2

MODELO FUERZA MOTRIZ - PRESIÓN - ESTADO - IMPACTO - RESPUESTA

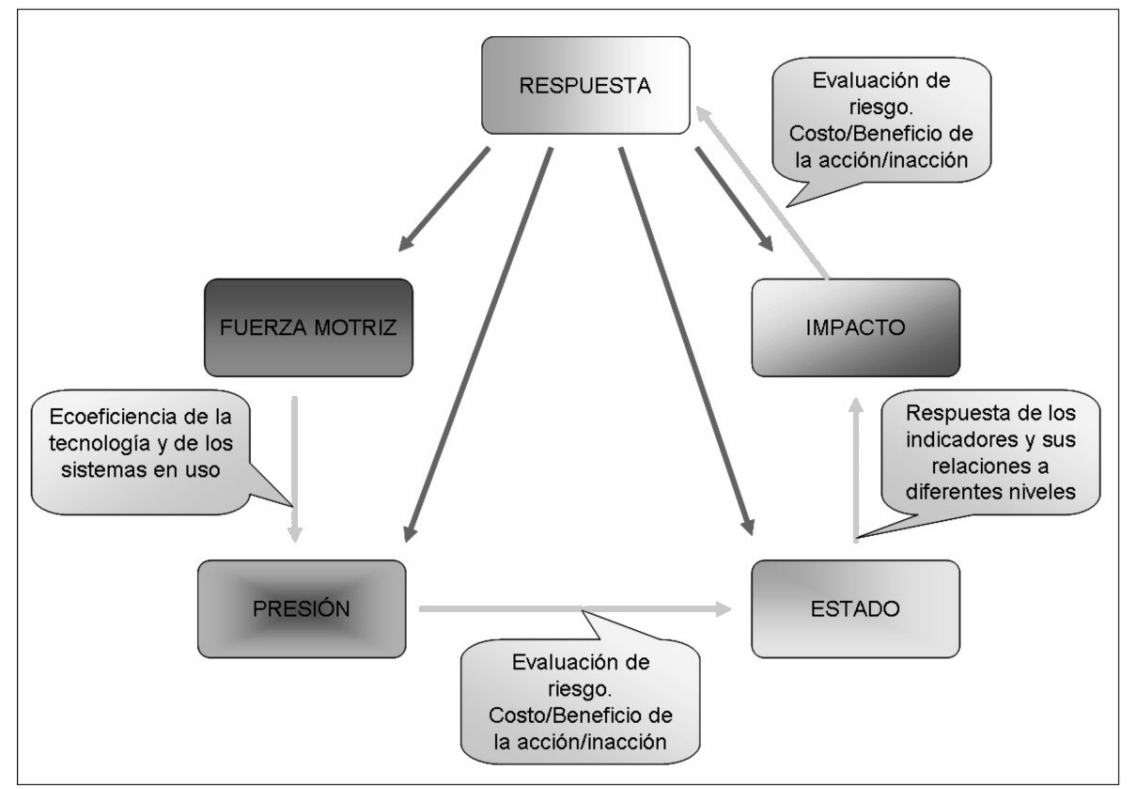

Fuente: Elaboración propia a partir de: AEMA, 2007.

Estudios Geográficos, Vol. LXXII, 271, pp. 611-654, julio-diciembre 2011

ISSN: 0014-1496, eISSN: 1988-8546, doi: 10.3989/estgeogr.201124 


\section{b) Sistemas de segunda generación}

Su utilización empezó en la década de los noventa, mediante el desarrollo de sistemas a nivel nacional, destacando las iniciativas realizadas por México, Chile, Estados Unidos, Reino Unido, España (OSE 2005 y 2006), etc. Se incorpora el enfoque multidimensional (económico, ambiental y social) del desarrollo sostenible. En los últimos años toma fuerza una cuarta dimensión, la institucional, debido a la relevancia e influencia que toman las políticas dictadas por los organismos de control (gobiernos locales, nacionales, organismos internacionales, etc.). El desarrollo de estos sistemas ha sido liderado por la Comisión de Desarrollo Sostenible de las Naciones Unidas, con indicadores que se engloban en cada una de las dimensiones del desarrollo, pero sin estar vinculados entre sí.

El Sistema de Indicadores de la Comisión de Desarrollo Sostenible-Naciones Unidas se puso en marcha para desarrollar el capítulo 40 de la Agenda 21 a través del «Programa de Trabajo en Indicadores de Desarrollo Sostenible», y produjo un listado de Indicadores de Sostenibilidad, desarrollados por hojas metodológicas, que fueron publicados en 1998 en el libro Indicators of Sustainable Development. Framework and Methodologies o también conocido como «Libro azul de los indicadores» (Quiroga, 2001). Inicialmente utilizó el marco ordenador FER hasta el año 2001 cuando desarrolla un nuevo marco ordenador basado en dimensiones, temas y subtemas (Naciones Unidas, 2001; CSD, 2006a; CSD, 2007; Quiroga, 2001).

En el año 2006 se reunió el Grupo de Expertos en Indicadores de Sostenibilidad para revisar la lista publicada en el año 2001, dando como resultado 14 temas, que agrupan los 96 indicadores desarrollados (Naciones Unidas, 2001; CSD, 2006b; CSD, 2007). Los Indicadores, aplicados en un ámbito geográfico nacional, han sido clasificados en básicos y no básicos, siendo los básicos aquellos que tienen importancia o son relevantes para la mayoría de países, mientras que los no básicos o complementarios proporcionan información adicional o se refieren a problemas que son relevantes en algunos países. Los 14 temas acordados son: Pobreza, Gobierno, Salud, Educación, Demografía, Riesgos Naturales, Atmósfera, Tierra, Océanos, Mares y Costas; Agua potable, Biodiversidad, Desarrollo Económico, Cooperación económica mundial y Patrones de consumo y producción (CSD, 2006 b).

Los criterios para la selección de indicadores son:

- Referirse a temas o cuestiones relevantes para el desarrollo sostenible en la mayoría de países. 
- Proporcionar información esencial no disponible de otros indicadores básicos.

- Pueden calcularse para la mayoría de los países con los datos existentes, o que podrían estar disponible en un plazo y costes razonables.

\section{c) Indicadores de tercera generación.}

En los últimos años, la necesidad de vincular las dimensiones del desarrollo y de sus indicadores entre sí, generó sistemas de indicadores que permitan tener un acceso rápido a un mundo de significados mucho mayor, y que los agrupe en temas o áreas multidimensionales, de forma transversal y sistemática (figura 3). Se destacan las iniciativas realizadas por la Unión Europea, a través del Grupo de Trabajo sobre Indicadores de Desarrollo Sostenible, y su adaptación en España por parte del Observatorio de la Sostenibilidad en España (OSE) en su informe 2007. Si bien se generaron a nivel internacional, los nuevos sistemas de indicadores propician un mayor componente territorial, que facilite su uso y aplicación a nivel local y promueva una mayor participación social.

FIGURA 3

\section{ENFOQUE TRANSVERSAL DE LOS SISTEMAS DE TERCERA GENERACIÓN}

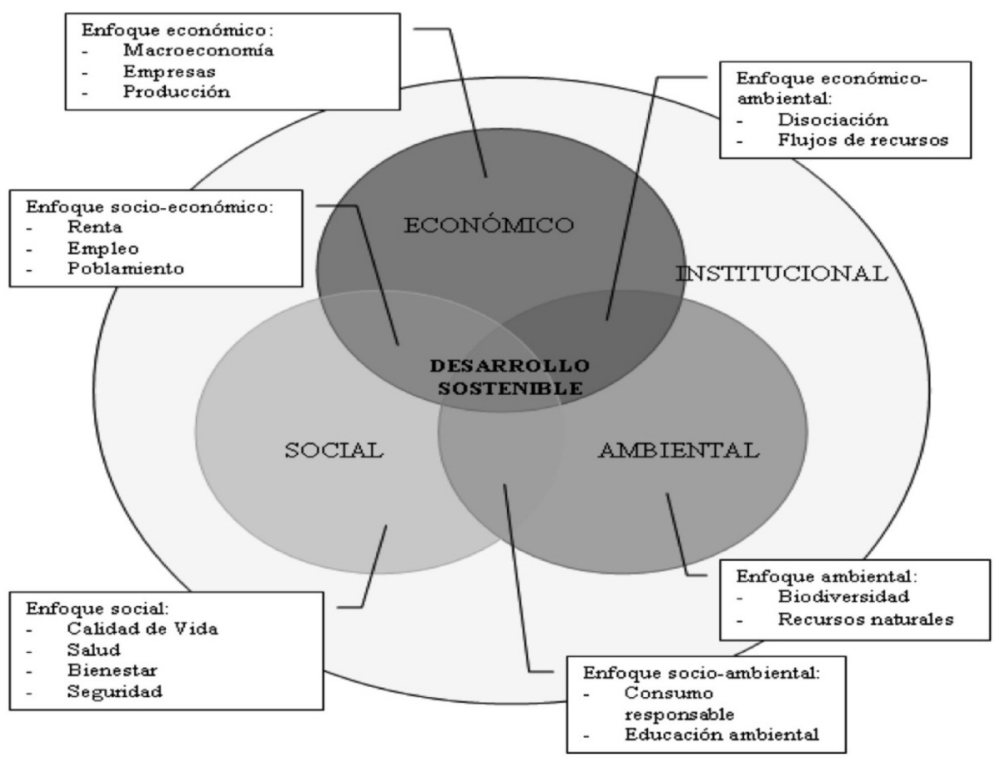

Fuente: Elaboración propia a partir de: Fernández, 2006; Gallopin, 2006; OSE, 2006. 
El Sistema de Indicadores de la Unión Europea fue creado bajo la consigna de mejorar la calidad de vida y el bienestar para las generaciones presentes y futuras, al amparo de la Estrategia de Desarrollo Sostenible, aprobada por el Consejo Europeo en junio de 2001 en Gotemburgo, y renovada en junio de 2006. En esta Estrategia, se establece desarrollar un Sistema de Indicadores para su seguimiento, bajo la responsabilidad de Eurostat. Aplica un sistema de indicadores de tercera generación, basado en diez temas, que reflejan los siete retos clave de la estrategia, el objetivo clave de la prosperidad económica, y los principios rectores en materia de buena gobernanza.

Para facilitar el manejo de los indicadores, y por ende mejorar su comunicación, el conjunto de indicadores se distribuye en una pirámide de tres niveles, que reflejan la estructura de la estrategia (objetivos generales, objetivos operativos y acciones), complementando la información con indicadores contextuales, que proporcionan información de los antecedentes de cada tema, pero que no inciden directamente sobre los objetivos de la estrategia (figura 4). Los indicadores son revisados cada dos años, determinando su funcionalidad y si responden a las necesidades de los países miembros de la UE.

FIGURA 4

ESTRUCTURA DEL SISTEMA DE INDICADORES DE LA UNIÓN EUROPEA

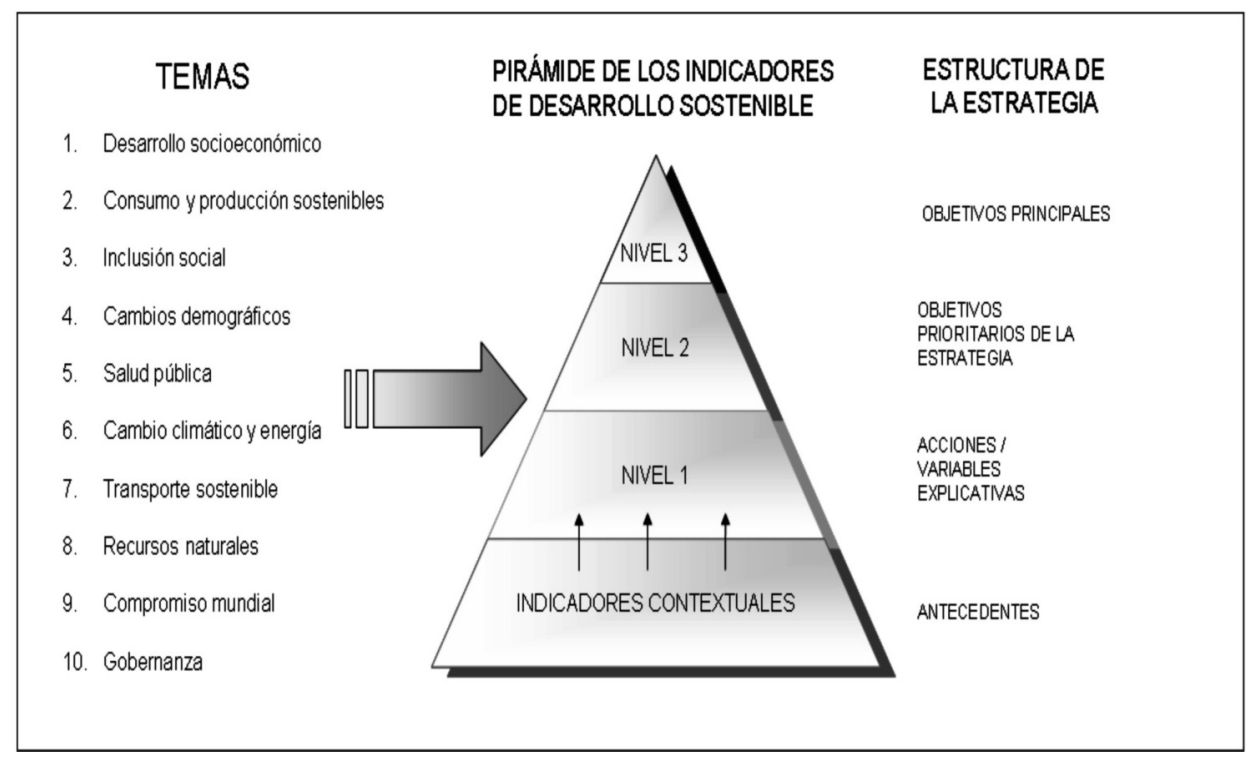

Fuente: Elaboración propia a partir de EUROSTAT, 2007. 
El Observatorio de la Sostenibilidad en España (OSE) ha publicado tres informes (2005, 2006 y 2007) que pretenden evaluar los procesos de sostenibilidad del desarrollo en España y analizar los logros y carencias actuales y previsibles (a nivel autonómico y nacional) con el objetivo de progresar hacia un desarrollo más sostenible. Responde a los requerimientos realizados por la Unión Europea a través de la llamada Agenda para el Cambio constituida por la revisada Agenda Socioeconómica de Lisboa y la Estrategia de Desarrollo Sostenible de Gotemburgo.

Adopta el marco FPEIR para los indicadores ambientales y su disociación de los sectores económicos. En cambio, los indicadores económicos y sociales son analizados de manera cualitativa y se pone de manifiesto su importancia dentro del desarrollo sostenible del país. El ámbito geográfico de aplicación del Sistema de Indicadores del Observatorio de la Sostenibilidad en España es nacional y regional (Comunidades Autónomas). En el informe del 2007 se adapta el marco temático de la UE, compuesto por diez áreas temáticas, a la realidad española, y se añade el área de Cultura y Desarrollo sostenible.

Los tres informes han mantenido la siguiente estructura de presentación:

- Objeto y metodología del informe, donde se analiza la sostenibilidad a nivel mundial, comunitario y nacional para definir los objetivos trazados por el OSE, y se presenta la metodología para obtener los indicadores desarrollados.

- Evaluación integrada, que contiene las conclusiones generales obtenidas del proceso de análisis de los indicadores desarrollados, poniendo especial énfasis en áreas de interés actual y político. Incluye un sistema de evaluación de cada uno de los indicadores desarrollados en cuanto a su estado actual y tendencias, valorándolos según el análisis de la ficha (progreso positivo o negativo, o situación indefinida o no evaluable).

El Marco de referencia, incluido en los informes de los años 2005 y 2006 que contextualizaba los procesos de desarrollo, identificaba las principales características del entorno físico y ambiental, junto con la estructura económica de los sectores productivos y la estructura administrativa de España, desapareciendo en los informes del 2007 y 2008, de tal forma que los temas tratados en ellos se incluyen en el ámbito de las áreas temáticas.

Así pues, a lo largo de estos cuatro años, el Observatorio de Sostenibilidad, ha modificado la organización de sus indicadores en áreas o sectores (en los dos primeros) hasta llegar a un marco ordenador por áreas temáticas (en los dos últimos), generando y aplicando nuevos indicadores y temas económicos, ambientales e institucionales (sirvan de ejemplo los de dependencia, sobrepeso, gasto social, parámetros macroeconómicos, transporte, buen gobierno, cultura...). 


\section{d) Consideraciones finales}

Los Sistemas de Indicadores de Sostenibilidad llevan a su haber cerca de treinta años de continuos cambios y avances hacia modelos interdimensionales que permitan evaluar de forma fácil y fiable los procesos de desarrollo. Los primeros modelos de Sistemas de Indicadores de Sostenibilidad, fundamentalmente de tipo ambiental y de carácter nacional o supranacional, han evolucionado hacia sistemas recientes que abordan la triple dimensión económica, ambiental y social de manera integral (figura 5).

Los sistemas internacionales (OCDE, ONU, UE) se han erigido como un importante referente para la elaboración de sistemas nacionales y regionales, sobre todo en los países desarrollados. Han desarrollado nuevos marcos ordenadores y han dictado las pautas a seguir para adaptarlos a otras escalas geográficas, y aportan gran cantidad de valores de referencia y procedimientos técnicos de cálculo de los indicadores. Generalmente, toman como valores objetivos aquellos que se han trazado en los distintos tratados, convenios u objetivos a nivel mundial (Protocolo de Kyoto, Objetivos del Milenio, Agenda 21, Estrategia de Gotemburgo, etc...) y se busca que los países tomen como guía sus indicadores para el desarrollo de Programas y Planes de Desarrollo Sostenible. Permiten ser desagregados a nivel sectorial y territorial, facilitando el enlace con otros sistemas y modelos. Pero para ello es necesario contar con datos suficientes y, actualmente, las bases de datos presentan varias deficiencias, especialmente en los temas ambientales y a nivel local, lo que dificulta el cálculo de los indicadores.

En España existen municipios que han implementado las Agendas 21 locales, basadas en el sistema de indicadores de la ONU, pero el uso del marco ordenador PER ha provocado que la mayor parte de indicadores sean de índole ambiental y mantengan la linealidad del sistema. Por otro lado no se aportan metas ni objetivos a mediano y largo plazo, como medida para llegar a la sostenibilidad. Además, es necesario generar indicadores de percepción en todas las dimensiones o áreas temáticas del sistema de indicadores, con el fin de evaluar la satisfacción de la población con el estado actual, de conocer el grado de aceptación de las actuaciones de los organismos públicos, y de buscar formas de comunicación que permitan una mayor proximidad a la sociedad en general y de forma particular a los gestores locales. Por último, las Agendas 21 han priorizado su trabajo en grandes ciudades, a nivel urbano, y en menor medida en los pequeños municipios rurales, de ahí el interés de la metodología que se propone. 


\section{FIGURA 5}

\section{EVOLUCIÓN DE LOS SISTEMAS DE INDICADORES DE SOSTENIBILIDAD}

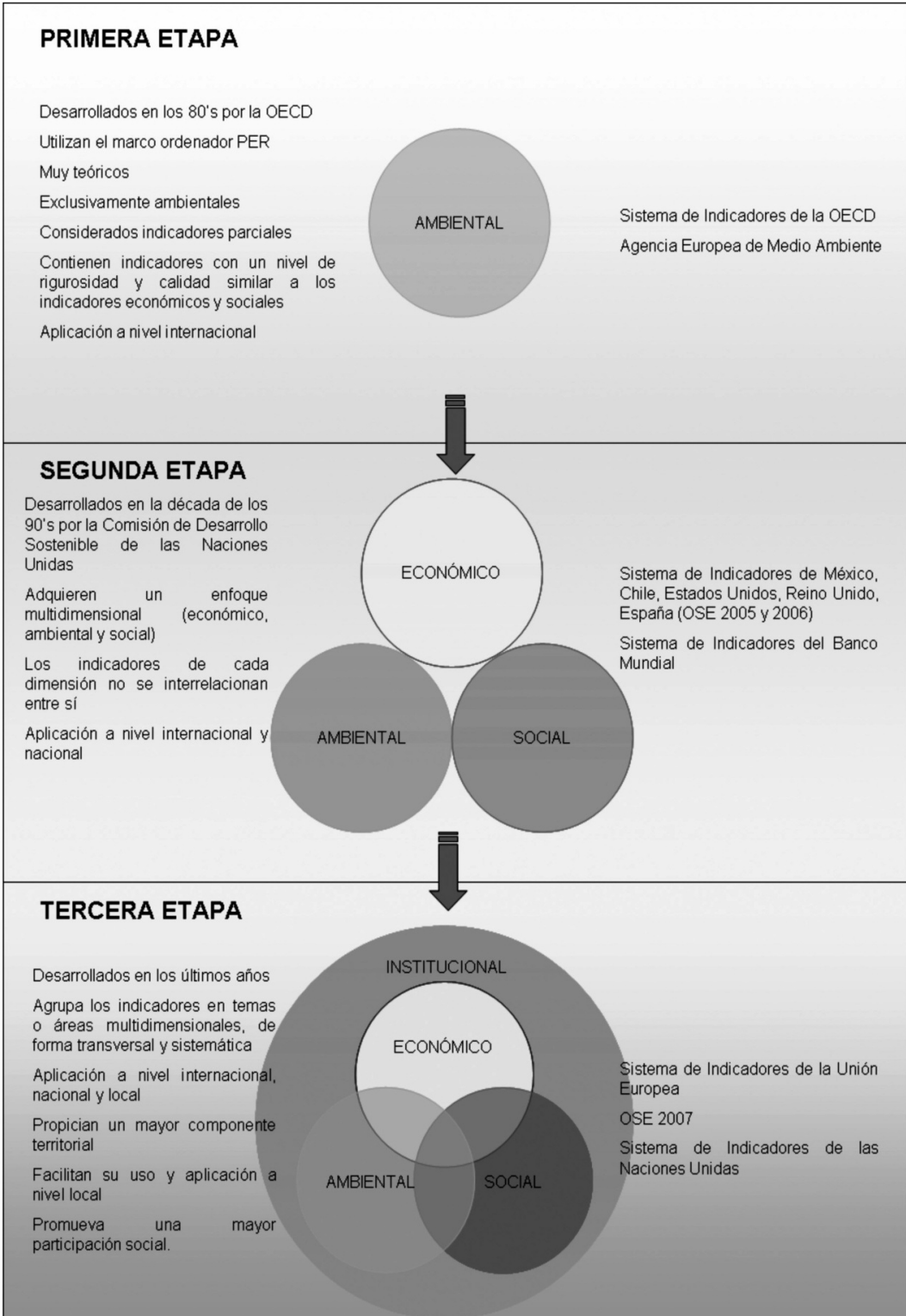

Fuente: Elaboración propia.

Estudios Geográficos, Vol. LXXII, 271, pp. 611-654, julio-diciembre 2011

ISSN: 0014-1496, eISSN: 1988-8546, doi: 10.3989/estgeogr.201124 


\section{Modelo DE CONSTRUCCIÓN DE UN SISTEMA DE INDICADORES DISEÑAdO PARA} ESPACIOS RURALES LEADER Y PRODER ESPAÑOLES

\section{Introducción a la metodología. Ciclo del indicador y metamodelo}

Reed, Frases y Dougill (2006) describen el proceso de desarrollo y aplicación de un Sistema de Indicadores de Sostenibilidad, mediante una aproximación top down-bottom up. Consta de cuatro etapas compuestas por doce fases que cierran un ciclo iterativo de evaluación de la sostenibilidad; es un marco de referencia empírico, en el cual se debe incluir las herramientas para su aplicación, de acuerdo con contexto de las áreas de estudio. En la figura 6 se observa el proceso o ciclo del indicador.

FIGURA 6

\section{CICLO DE DESARROLLO Y APLICACIÓN DE UN SISTEMA DE INDICADORES}

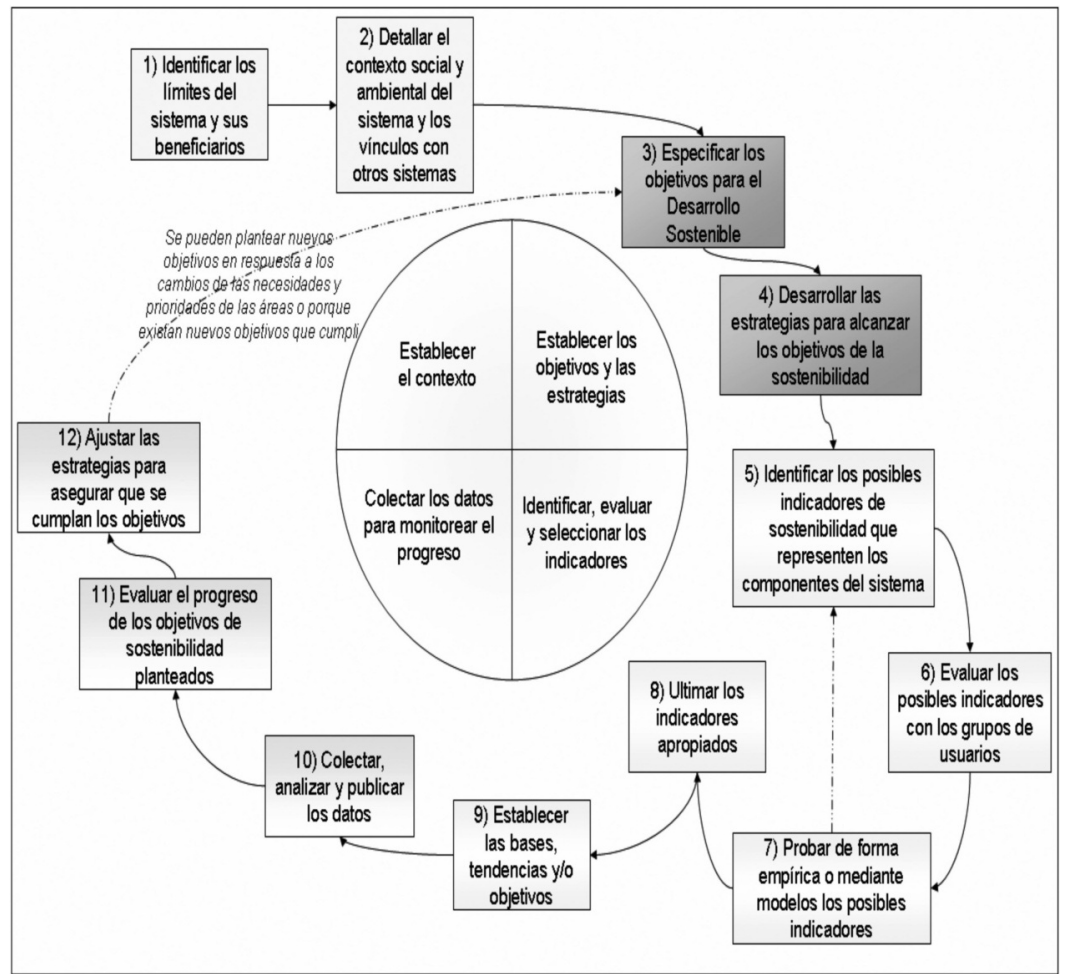

Fuente: Reed, Frases y Dougill, 2006. 
Se propone un Metamodelo, o modelo de modelos, de construcción de un Sistema de Indicadores de Sostenibilidad de Procesos de Desarrollo en espacios territoriales, que consta de ocho fases iterativas. El planteamiento permite adaptarlo a distintas escalas, a distintos sectores, y a enfoques bottom-up y top-down. Las fases generales del metamodelo se agrupan de acuerdo con la siguiente estructura:

TABLA 1

FASES DEL METAMODELO DE CONSTRUCCIÓN DE UN SISTEMA DE INDICADORES DE SOSTENIBILIDAD

1. CONTEXTO DEL SISTEMA DE INDICADORES

1.1. Ámbito Geográfico del Sistema de Indicadores

1.2. Enfoque sectorial del Sistema de Indicadores

1.3. Contexto Político-Administrativo

1.4. Selección de Periodos cronológicos claves

2. TRABAJOS DE INVESTIGACIÓN PREVIOS

2.1. Caracterización de Sistemas de Indicadores existentes de utilidad

2.2. Caracterización de las Bases de Datos útiles

3. PROCESO DE GENERACIÓN DE INDICADORES

3.1. Estructura inicial del Sistema de Indicadores. Clasificación. Áreas temáticas prioritarias

3.2. Generación de Indicadores simples por áreas temáticas

3.3. Generación de Indicadores complejos. Estructura final del Sistema de Indicadores.

4. PROCESO DE SELECCIÓN DE INDICADORES

4.1. Caracterización y Valoración de Indicadores

4.2. Selección de indicadores

5. CONSTRUCCIÓN Y APLICACIÓN DE LOS INDICADORES SELECCIONADOS

5.1. Características, construcción técnica y selección de los indicadores relativos

5.2. Datos necesarios para el cálculo de los indicadores relativos

5.3. Cálculo y aplicación de los indicadores en las áreas geográficas.

6. DESARROLLO DEL SISTEMA DE INDICADORES

6.1. Valores de Referencia.

6.2. Estimación de los Valores Umbrales: críticos y deseables.

6.3. Estimación de los Valores Objetivos de aproximación hacia el Valor Deseable

7. DISCUSIÓN E INTERPRETACIÓN DE LOS RESULTADOS

7.1 Análisis temático: análisis por áreas temáticas en el conjunto de la red

7.2. Análisis geográfico: análisis de todo el sistema de indicadores en cada área geográfica

8. COMUNICACIÓN DEL SISTEMA DE INDICADORES

8.1 Estructura de la información de cada indicador y divulgación

8.2 Uso del Sistema de Indicadores.

Fuente: Elaboración propia.

Estudios Geográficos, Vol. LXXII, 271, pp. 611-654, julio-diciembre 2011

ISSN: 0014-1496, eISSN: 1988-8546, doi: 10.3989/estgeogr.201124 
A continuación, se exponen las características del metamodelo, y se realiza la adaptación a los procesos de desarrollo en áreas rurales españolas Leader y Proder. En este caso, el principal objetivo del Sistema de Indicadores es ofrecer a los gestores de los programas comarcales de desarrollo rural y a la población local las herramientas necesarias para la toma de decisiones.

\section{Contexto del sistema de indicadores}

El contexto en el que se enmarcan los procesos y las dinámicas derivadas de las interacciones socioeconómicas y ambientales, de acuerdo con las características biogeográficas y socioeconómicas del área de estudio, determinará el cumplimiento de los objetivos del Sistema de Indicadores (OSE, 2006). Por eso es necesario definir, en primer lugar, el contexto de los mismos, en particular el ámbito geográfico, el enfoque sectorial, el tipo de desarrollo, el contexto político-administrativo y los periodos cronológicos de evaluación.

\section{1. Ámbito geográfico del sistema de indicadores}

Teniendo en cuenta que la sostenibilidad de un sistema debe procesarse en la globalidad del sistema, no en la de sus dimensiones, se entiende y se acepta el estudio de la sostenibilidad atendiendo a diferentes escalas espacio-temporales y dimensiones de análisis (Fernández, 2006). Cada nivel de escala posee diferentes problemas, cuestiones a resolver y teorías a formular. Por tanto, la forma de comprender y analizar los fenómenos tiene valor cuando se los colocan en escalas de magnitud comparables, aun cuando el estudio de las transferencias entre distintas escalas revierte interés.

La clasificación más extendida establece los siguientes ámbitos: Internacional, Nacional, Regional y Local. De acuerdo con la realidad española, se pueden establecer los siguientes niveles de escalas:

-Internacional:

- Mundial

- Continental (UE,...)

- Nacional y Regional:

- Nacional

- Comunidades Autónomas 
- Provincial

- Local:

- Comarcal

- Municipal (Agenda 21 local)

Fraser et al. (2005) sostienen que la elección del ámbito geográfico es determinante para la construcción del sistema y su aplicación. Consideran que la provincia resulta un ámbito geográfico muy grande para ser significativa, porque no analiza las variaciones locales existentes, que resultan importantes para el desarrollo de un sistema de indicadores. En cuanto a la comarca, consideran que se debe evitar que los datos obtenidos escondan las tendencias reales de los indicadores y conduzcan a la duplicación de los servicios y a una falta de armonización de la región. También es necesario, en el momento de construir los indicadores, especificar su ámbito o ámbitos unitarios más frecuentes, generalmente determinados por el alcance de la base de datos o el trabajo de campo utilizado (Bakkes et al., 1994; OSE, 2005; Reed, Frases y Dougill, 2006).

El ámbito geográfico de un Sistema de Indicadores estará determinado por la estructura político-administrativa de las áreas sobre las que se aplica. En consecuencia, el ámbito geográfico del sistema de indicadores para áreas rurales Leader y Proder de España debe ser el comarcal, y el ámbito unitario más frecuente, aunque éste dependerá de la disponibilidad de datos del indicador, es el término municipal.

\subsection{Enfoque sectorial del análisis}

Una vez delimitado el ámbito geográfico, conviene determinar los sectores principales y las actividades preponderantes de desarrollo de las áreas analizadas, o el tipo de desarrollo principal del análisis, al objeto de orientar la naturaleza del sistema de indicadores. El enfoque sectorial dependerá del objetivo del sistema de indicadores, y en la actualidad se pueden citar como ejemplos los sistemas desarrollados para los sectores Turístico, Urbano, Agrícola intensivo, Industrial, Energético, de la Atmósfera, del Transporte, Forestal, para Costas y medio marino, Cuencas Hidrográficas, Suelos y Residuos, de aplicación de la Agenda 21, etc.

De acuerdo con las características de las comarcas rurales Leader y Proder españolas, el enfoque sectorial es claramente de tipo rural extensivo o de montaña. La aplicación de los Indicadores en las áreas rurales nos permitirá 
extraer conclusiones sobre la sostenibilidad de los Procesos de Desarrollo y evaluar la efectividad de las políticas de desarrollo rural de la UE, apoyándonos en los resultados en estas áreas piloto.

\subsection{Contexto Político-Administrativo}

Una vez determinados el ámbito geográfico y el enfoque sectorial del Sistema de Indicadores, el metamodelo considera necesario establecer el contexto político-administrativo. Éste influirá sobre el análisis de los indicadores porque reflejan elementos externos al Sistema, que condicionan su actividad y resultados. Las áreas rurales españolas se corresponden con el contexto político-administrativo de gestión descentralizada por las comunidades autónomas, coordinada por el estado español e insertado en el contexto de la Unión Europea, organismo que determina las políticas y directrices de desarrollo rural que son aplicadas en las áreas españolas.

\subsection{Selección de periodos cronológicos claves}

Bossel (1999) expresa que el desarrollo sostenible implica cambios constantes, y los sistemas de indicadores por sí mismos tienen que adaptarse a las nuevas condiciones. Es por lo tanto necesario establecer periodos cronológicos claves fundamentales de análisis, a partir de hitos, hechos o acontecimientos claves, tanto en el área como en el sector en el que se inserta el sistema de indicadores. Al menos se deberán establecer los siguientes periodos: pasados para la evaluación ex-post, actuales o pasados recientes, y futuros para la evaluación ex-ante. En definitiva, se analizará la evolución histórica de los indicadores, que permitirá su evaluación diacrónica para tener una perspectiva global y conocer sus tendencias.

En el caso de las áreas rurales Leader y Proder, se ha fijado el periodo 19912001 como periodo cronológico pasado, por coincidir con las primeras políticas de desarrollo rural en la UE, en particular la iniciativa Leader I, Leader II, y primeros Proder. La evaluación ex-post de este periodo permitirá, como indica González (2000): «Una valoración a posteriori sobre la pertinencia, eficacia, eficiencia y efectos del proyecto en el contexto de sus objetivos declarados».

El periodo actual o pasado reciente abarca el periodo 2002-2006, y permitirá la evaluación de la sostenibilidad del proceso actual de desarrollo. Se fijó este periodo por coincidir con las políticas recientes de desarrollo rural, en particular la iniciativa Leader+y los Proder II. 
Como periodo futuro, se fija el periodo 2007-2013, que es objeto de las nuevas políticas de desarrollo rural determinadas por la Unión Europea, que se centran en tres ámbitos que corresponden a tres ejes temáticos del nuevo Reglamento: mayor competitividad del sector agrario y forestal, mejora del medio ambiente y el entorno rural y mejora de la calidad de vida en las zonas rurales y diversificación de la economía rural y se apoya en cuatro ejes, siendo uno de ellos el Leader, compatible con los otros tres (Comunidades Europeas, 1995-2007). En este periodo se analizarán las tendencias, con el objeto de determinar y contrastar los valores:

- Futuros (por estimaciones estadísticas)

- Umbrales (críticos y deseables)

- Posibles (u objetivos de progreso realista hacia los valores deseables)

TABLA 2

CONTEXTO DEL SISTEMA DE INDICADORES PARA ÁREAS RURALES ESPAÑOLAS

\begin{tabular}{ll}
\hline Ámbito Geográfico & - Ámbito total: Comarcal \\
& - Ámbito unitario: Municipal (generalmente) \\
\hline Enfoque sectorial & De tipo rural extensivo o de montaña \\
\hline Contexto político-administrativo & - Directrices Europeas \\
& - Coordinación Estatal \\
& - Gestión por las Comunidades autónomas \\
\hline Periodos de análisis diacrónico & - Pasado: 1991-2001 \\
& - Reciente: 2002-2006 \\
\hline
\end{tabular}

Fuente: Elaboración propia.

Una vez fijado el contexto del Sistema de Indicadores, resumido en la tabla 2 , se recomienda recurrir a un ciclo iterativo (figura 7), que consta de los pasos fundamentales siguientes:

- Generación de posibles indicadores.

- Selección de los indicadores óptimos.

- Construcción y aplicación de los indicadores seleccionados.

- Desarrollo del Sistema de Indicadores (valores umbrales y objetivos).

- Comunicación, transferencia y uso de los Indicadores.

- Generación de nuevos Indicadores... 


\section{FIGURA 7}

CICLO ITERATIVO DE LA CONSTRUCCIÓN DE UN SISTEMA DE INDICADORES

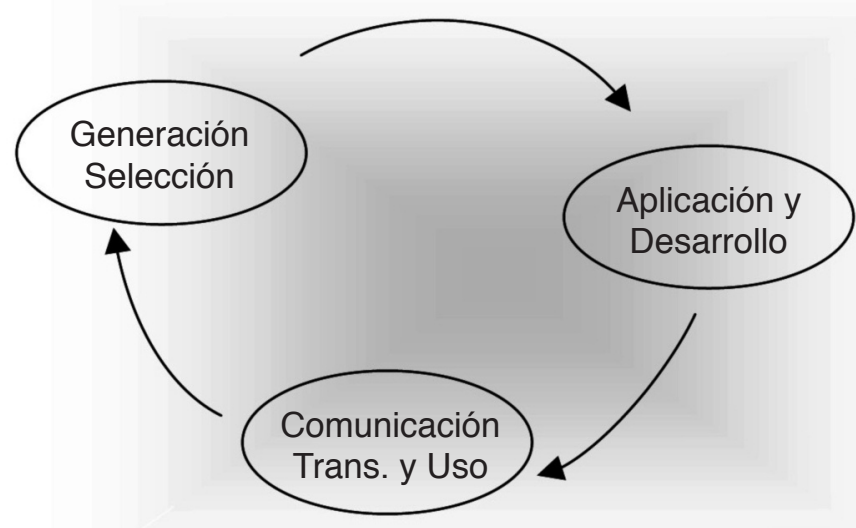

Fuente: Elaboración propia.

\section{Trabajos de investigación previos}

\subsection{Caracterización de Sistemas de Indicadores existentes de utilidad}

Se analizarán, clasificarán y caracterizarán los Modelos de Indicadores disponibles en la bibliografía, relacionados con el Sistema que se pretende definir y desarrollar. Éstos nos servirán en la fase de generación de indicadores y como valores de referencia para analizar los resultados obtenidos.

\subsection{Caracterización de las Bases de Datos útiles}

Una base de datos o banco de datos es un conjunto organizado de datos o valores estadísticos que pertenecen al mismo contexto, almacenados sistemáticamente para facilitar su uso. Para recopilar datos de fuentes de información primaria y secundaria, se precisa analizar, clasificar y caracterizar cada una de las bases de datos disponibles, en particular:

- Información bibliográfica.

- Registros administrativos procedentes de diversas administraciones. 
- Mediciones técnicas y estadísticas realizadas en trabajos científicos.

- Información cartográfica y analógica.

En la construcción del sistema de indicadores es necesario recopilar toda la información disponible, principalmente de la escala de aplicación, y sistematizarla, para lo cual es importante conocer, para cada base de datos, la estructura, los datos disponibles, en particular de sus ámbitos total y unitario: municipio, provincia,... y las series de años disponibles.

Finalmente, se deberá realizar una valoración general del conjunto de las Bases de Datos: calidad y fiabilidad, facilidad para operar..., con el objeto de conocer los déficits detectados y realizar propuestas de búsqueda y obtención de datos complementarios a través de otras fuentes: direcciones de correos electrónicos y/o teléfonos de administraciones, empresas, etc.

\section{Proceso de generación de indicadores}

\subsection{Estructura inicial del Sistema de Indicadores en Áreas Temáticas}

La estructura inicial de un sistema de indicadores debe establecerse bajo una clasificación que permita la especialización por áreas temáticas en la generación inicial de indicadores y en el análisis de la sostenibilidad económica, ambiental y social del proceso de desarrollo. La estructura debe tener como base la identificación de los diferentes sectores o áreas temáticas, así como los sistemas sobre los que depende la sociedad humana (Bossel, 1999).

Se propone como primera clasificación general de los indicadores un sistema compuesto por cuatro grandes subsistemas: medio económico, medio natural, medio social y un marco de referencia, que incluye los temas institucionales, para caracterizar cada una de las áreas de estudio que permita el análisis adecuado de los resultados obtenidos.

Se propone realizar una primera desagregación en medios y bloques. El medio corresponde al primer nivel de desagregación, de acuerdo con los tres principios básicos de la sostenibilidad: económica, ambiental y social; y el bloque, al segundo nivel, con posibilidad de matizar, en su caso, en subbloques o subgrupos. Así, obtendríamos, con carácter genérico:

- Marco de Referencia natural y antrópico (que incluye el institucional).

- Medio Económico.

- Sectores productivos (primario, secundario y terciario)

- Sectores auxiliares (energía, transporte y equipamientos)

- Estructura económica 
- Medio Natural.

- Suelo, agua, atmósfera

- Biodiversidad: vegetación y fauna

- Costas y medio marino

- Medio Social.

- Población. Estructura demográfica. Poblamiento

- Economía de la población. Mundo laboral

- Bienestar social

Posteriormente se puede desglosar la estructura en conceptos, perfiles e indicadores, donde el Concepto corresponde al tercer nivel de desagregación, con posibilidad de matizar, en su caso, en subconceptos, el Perfil corresponde al cuarto nivel de desagregación, con posibilidad de matizar, en su caso, en subperfiles, y el Indicador corresponde al último nivel de desagregación. El desglose progresivo de la estructura del sistema de Indicadores en áreas temáticas se realiza en función de su naturaleza, principalmente del tipo de desarrollo.

Aplicando estas directrices generales a las comarcas de desarrollo rural, la clasificación adaptada deberá hacer mayor énfasis en el suelo: residuos, erosión,..., en la utilización y la calidad del agua y en las aguas residuales, en la biodiversidad, especialmente la gestión forestal y de los ENP, en la participación social dado el carácter local, en los sectores productivos ligados a la agricultura y ganadería y en el turismo rural. Por otro lado, se suprime el apartado de costas y medio marino. De esta forma, la estructura inicial quedaría de la siguiente manera:

TABLA 3

ESTRUCTURA INICIAL DEL SISTEMA DE INDICADORES EN ÁREAS TEMÁTICAS, APLICADA PARA ESPACIOS RURALES

\section{A. MARCO DE REFERENCIA}

1. CONTEXTO. Administrativo: superficies, población. Características físicas y climáticas (altitud...). Instituciones.

2. USOS. Superficies de usos del suelo (Naturales y Antrópicos).

\section{B. MEDIO ECONÓMICO Y SECTORES PRODUCTIVOS. SOSTENIBILIDAD ECONÓMICA}

1. SECTORES PRODUCTIVOS CON BASE AGRARIA

A. Agricultura. Estructura. Agricultura ecológica.

B. Ganadería. Vías pecuarias. Alimentación animal. Aspectos económicos, sociales y ambientales.

C. Industria agroalimentaria. Aspectos económicos, sociales y ambientales.

D. Sanidad y Seguridad alimentaria. 
2. SECTORES PRODUCTIVOS NO AGRARIOS

A. Turismo. Recursos. Oferta. Demanda. Aspectos económicos, sociales y ambientales.

B. Otros sectores. Doméstico. Industria no agroalimentaria. Comercio. Artesanía. Servicios. Actividades extractivas y mineras. Construcción. Aspectos económicos, sociales y ambientales.

3. ESTRUCTURA ECONÓMICA DEL ÁREA

A. Parámetros macroeconómicos: PIB, sectores productivos...,

B. Estructura empresarial. PyMES y familiares. Calidad y Gestión integrada. Tecnología. Competitividad. Impuestos.

4. SECTORES AUXILIARES

A. Energía. Primaria. Alternativas. Final. Aspectos económicos, sociales y ambientales.

B. Transporte. Infraestructura. Actividad. Aspectos económicos, sociales y ambientales.

\section{MEDIO NATURAL. SOSTENIBILIDAD AMBIENTAL}

1. SUELO

A. Calidad del suelo. Degradación. Contaminación. Erosión. Riesgos: inundación, sísmico.

B. Residuos. Generación. Gestión. Materiales.

2. AGUA

A. Aguas superficiales y subterráneas. Recursos y explotación. Gestión. Precio del agua. Calidad del agua: contaminación, salinización. Gestión de la calidad del agua.

B. Aguas residuales: generación y gestión.

\section{ATMÓSFERA}

A. Calidad del Aire. Contaminación. Salud ambiental. Contribución a la contaminación global y el cambio climático.

\section{BIODIVERSIDAD}

A. Biodiversidad. Espacios Naturales. Espacios forestales. Degradación. Gestión. Incendios.

B. Flora y Fauna. Especies. Endemismos. Hábitats faunísticos.

C. Espacios Naturales Protegidos. Superficies. Presiones. Planificación de ENP. Gestión.

\section{MEDIO SOCIAL. SOSTENIBILIDAD SOCIAL}

1. POBLACIÓN

A. Demografía. Estructura. Natalidad. Envejecimiento. Migraciones.

2. ECONOMÍA DE LA POBLACIÓN

A. Renta. Mundo laboral. Población Activa. Paro. Seguridad laboral.

3. BIENESTAR SOCIAL

A. Salud. Servicios públicos sociales. Salud ambiental.

B. Educación. Cultura. Deporte. Cohesión social.

C. Democracia local y participación social. Educación ambiental. Igualdad de género. 4. POBLAMIENTO, VIVIENDA Y URBANISMO.

A. Poblamiento. Pequeños núcleos urbanos. Urbanismo. Vivienda en el medio rural.

B. Vertebración Territorial.

\section{E. OTROS INDICADORES: TRANSVERSALES, INTEGRADOS, DE BALANCES...}

A. Fortaleza Institucional.

B. Ecoeficiencia. Disociación. etc.

Fuente: Elaboración propia. 


\subsection{Generación de indicadores simples por áreas temáticas}

El proceso de generación de indicadores es eminentemente creativo y se basa en la motivación, la información y la flexibilidad. En base a estos criterios, en el desarrollo de esta etapa, en primer lugar se deben recoger todos los indicadores propuestos en los modelos analizados, evitando redundancias. En segundo lugar, definir los indicadores a partir de los datos disponibles identificados, y finalmente, generar nuevos indicadores como consecuencia de una tormenta de ideas (se recomienda que esta fase final sea realizada por un equipo multidisciplinar para abarcar la mayor cantidad de enfoques posibles).

Bossel (1999), considera que un sistema viable de indicadores de sostenibilidad debe articularse en subsistemas (o niveles de desagregación) de forma que cada uno de ellos sean también viables. Por consiguiente, la tarea fundamental será encontrar los indicadores adecuados o idóneos para cada subsistema. Por otra parte, debemos identificar los indicadores que proporcionan información acerca de la contribución de cada subsistema a la viabilidad del sistema.

En un inicio, la generación de los indicadores debe partir de la clasificación establecida en la estructura inicial, es decir, especializándose por áreas temáticas. El Área temática se concibe como una parte más o menos amplia de la clasificación de indicadores, y no necesariamente tendrá que coincidir con un medio, grupo o concepto. Su definición será en función de su importancia y su carácter homogéneo para su estudio de forma inicialmente aislada. Reed, Frases y Dougill (2006) consideran que la generación e identificación de indicadores potenciales a menudo se encuentra a cargo de investigadores y responsables de formular políticas (top-down), debiendo ser necesario una aproximación bottom-up, mediante la inclusión de las partes interesadas, ya sea en la fase de generación o en la evaluación de los indicadores potenciales de la lista profusa.

\subsection{Generación de indicadores complejos o sintéticos. Estructura final del} Sistema de indicadores

Posteriormente, se generarán, a partir de los resultados de la aplicación de los indicadores simples, nuevos indicadores complejos o índices que permitan el análisis de la sostenibilidad de forma más global. De esta forma, se generarán áreas temáticas complejas.

En la construcción de índices o indicadores sintéticos de sostenibilidad existe una pérdida de información al agregarla o ponderarla en un solo valor; 
generalmente se desestima esta pérdida, dado el grado de avance hacia el objetivo del desarrollo sostenible en términos generales (Castro, 2004). Existen varios inconvenientes en la construcción de índices, principalmente los derivados de la heterogeneidad de los mismos, así como la simplificación excesiva, lo cual dificulta el poder recoger todas las interrelaciones entre los subsistemas (Gallopín, 1997). También pueden existir problemas de inconmensurabilidad y pérdida de información al sintetizar la misma. Para reducir el impacto de estos problemas, se utilizan las técnicas multicriterio en la ponderación y agregación de información multidimensional (Munda et al., citados por Castro, 2004).

Debido a la subjetividad presente en los métodos de ponderación, es necesario que estén relacionadas las reglas de agregación de los indicadores simples con las reglas que definen las interrelaciones entre el conjunto de indicadores seleccionados, constituyendo un auténtico modelo de la realidad (Castro, 2004). Gallopín (1997) refuerza este concepto al proponer que los indicadores seleccionados representen variables o propiedades del sistema completo, es decir, indicadores holísticos.

Al utilizar ponderaciones se puede diferenciar entre:

a) La distancia cuantificada entre el indicador simple y su valor de referencia (se consideran cuatro las posibles referencias: el nivel objetivo marcado por la política hacia la sostenibilidad, el nivel máximo/mínimo observado, el valor umbral o el nivel medio observado).

b) La opinión de expertos o bien el reflejo de las preferencias sociales en base a algún tipo de encuesta (Castro, 2004).

La bibliografía recoge varios métodos para la generación de índices:

- Agregación objetiva o matemática, mediante técnicas de análisis factorial.

- Superposición gráfica de los indicadores.

- Medida directa de los componentes de los indicadores con pesos implícitos en el modelo; $y$,

- Selección de ponderaciones exógenas sobre la base de determinadas hipótesis.

En definitiva, a partir de la estructura de los Indicadores por áreas temáticas de la primera generación, el proceso de generación de Indicadores avanzará de acuerdo con una estructura más compleja y culminará con la propuesta de Índices de Síntesis mediante agregación ponderada de Indicadores que facilite el análisis de la sostenibilidad de procesos de desarrollo de forma más global. 
De esta forma, se obtendrán áreas temáticas complejas, cuyo objetivo es englobar todas las dimensiones de la sostenibilidad, como por ejemplo: Desarrollo Socioeconómico, Sostenibilidad financiera pública, Innovación y Competitividad, Inclusión social, Demografía, Envejecimiento Demográfico, Salud Pública, Medio ambiente y Salud, Cambio climático y efecto invernadero, Energía, Producción y consumo sostenible, Ecoeficiencia, Gestión de los recursos naturales, Transporte, Buen Gobierno, Cultura, Bienestar social, Seguridad alimentaria, etc...

\section{Proceso de selección de indicadores}

\subsection{Caracterización}

Para iniciar la selección de los indicadores, primero deben definirse los grupos de atributos que servirán para su caracterización. Se recomienda adaptar los atributos recomendados por la literatura especializada, para posteriormente aplicarlos al sistema que se desea construir. Se propone agrupar los atributos en tres grupos considerados básicos para la selección de Indicadores y la construcción del Sistema de Indicadores:

- Objetivos del Sistema de Indicadores.

- Calidad de los datos necesarios para el indicador.

- Interés para la sociedad.

Los principales atributos a considerar pueden ser:

Sobre los objetivos del Sistema de Indicadores:

- Evaluación de la sostenibilidad. El indicador contribuye a la evaluación de la sostenibilidad del desarrollo desde los puntos de vista económico, ambiental y/o social; y ayuda a la consolidación de los procesos institucionales de cambio.

- Objetivos del sistema. Asimismo, permite evaluar los efectos de las políticas sectoriales a las que pertenece el área de estudio.

- Significativo. Debe ser relevante y no redundante frente a otros similares, del mismo tipo o grupo.

- Cobertura geográfica. Es válido para el ámbito geográfico del sistema de indicadores considerado, en este caso, el local.

- Integración. A pesar de lo expresado anteriormente, este atributo permite que los indicadores puedan ser integrados en otros Sistemas de Indicadores. En este sentido, se distingue la integración: 
- Vertical, esto es susceptible de integrarse directa o indirectamente en sistemas superiores (nacionales, UE...) o inferiores: (agenda 21 municipales...)

- Horizontal, esto es susceptible de integrarse directa o indirectamente, para el mismo ámbito geográfico considerado, en otros sistemas sectoriales: turismo, agrícola...

Sobre la calidad de los datos necesarios para el Indicador:

- Disponibilidad. Se valora el que exista disponibilidad de datos en todas las áreas de estudio, y en todos sus niveles, y que existan valores de un periodo suficiente de años, actuales y pasados.

- Coste razonable. Prima la obtención del dato dentro de un ratio coste/beneficio razonable.

- Fiabilidad y consistencia metodológica. La metodología de obtención de los datos se deberá contrastar y determinar las fuentes seguras.

Relacionados con la Sociedad

- Interés social. El indicador deberá ser de interés social.

- Impacto y resonancia. Es decir considerado importante, urgente o de especial interés por la sociedad y las administraciones.

- Comprensible. De fácil comprensión por personas no expertas.

- Comunicación. Transmite de forma clara y amigable su contenido e importancia (las fichas resúmenes son una forma de comunicación de los indicadores).

- Metas. Permite la propuesta de metas futuras a alcanzar en sucesivos proyectos.

\subsection{Valoración}

La valoración de los indicadores simples y complejos requiere un proceso de análisis de sus atributos y valoración, por áreas temáticas, mediante encuestas y entrevistas a expertos.

A cada experto entrevistado se le explicará en primer lugar el contenido global del Sistema de Indicadores y sus objetivos, el objetivo concreto de la encuesta, y las características de los grupos de atributos o criterios de valoración de los indicadores.

Posteriormente, se le presentará la lista de indicadores generados, que podrá ser ampliada con nuevos indicadores propuestos por el experto, y se le pedirá que haga una preselección, eliminando los indicadores que considere que 
deban ser excluidos de la valoración por tener una valoración muy baja en uno o varios de sus atributos (porque no tengan ninguna validez desde el punto de vista de la evaluación de la sostenibilidad, porque no se disponen de datos de calidad, o porque no sean de ningún interés para la sociedad).

Sobre esa lista, se le pedirá que realice, para cada indicador, una valoración de 1 a 5 de acuerdo con cada grupo de atributos. Si lo prefiere, y al objeto de no alargar excesivamente el proceso, podrá realizar una valoración global de 1 a 5. Si ha optado por el primer caso, se tomará como valor final la media aritmética de las valoraciones. La escala de valores será adaptada a la realidad del medio, permitiendo variaciones de un grupo de indicadores a otro.

\subsection{Selección de Indicadores}

Se contemplan dos etapas para la selección de indicadores:

- Selección por áreas temáticas

En cada área temática, a partir de las caracterizaciones y valoraciones anteriores, se seleccionará un conjunto de indicadores que conformarán los subsistemas de indicadores correspondientes a las distintas áreas temáticas.

- Selección para todo el sistema de indicadores A partir de todos los subsistemas de indicadores de la primera fase, se seleccionará un conjunto completo de indicadores simples y complejos, que cubra todas las áreas temáticas, y equilibrado, con un número adecuado de indicadores y una distribución por áreas temáticas acorde a su importancia.

La lista de indicadores seleccionados será depurada mediante un proceso de caracterización y posterior valoración mediante atributos por parte de expertos y los actores involucrados en la construcción del sistema de indicadores (actores políticos, gestores, sociedad, etc.).

\section{Construcción técnica y aplicación de los indicadores}

\subsection{Características y construcción técnica de los indicadores}

En primer lugar, se pondrán de manifiesto todas aquellas características que describen las principales cualidades del indicador, entre los que destacamos su definición, su significado y justificación, su clasificación, su interacción y relación con otros indicadores, y su grado de madurez. 
La construcción técnica de los indicadores permite cuantificarlos para realizar la evaluación de la sostenibilidad, en particular para poder efectuar las comparaciones a diferentes escalas (internacional, nacional, regional, local) en un año determinado (comparación sincrónica) y para analizar las tendencias del desarrollo en el tiempo (comparaciones diacrónicas). El contar con una base de datos completa y actualizada, permitirá que la cuantificación del indicador aporte resultados reproducibles y analizables (Bossel, 1999).

La construcción técnica del indicador se realizará de forma que éste cumpla una serie de características:

- Consistencia metodológica en la construcción del indicador.

- Predictivo es decir sensible a los cambios de tendencia.

- Comparable, debiendo permitir las comparaciones diacrónicas, esto es, a lo largo del tiempo, y sincrónicas, entre distintas áreas geográficas (términos municipales, comarcas, provincias, comunidades, países, etc), en fechas claves.

Para que el indicador cuantificado sea comparable y predictivo, deberá expresarse en términos relativos, utilizando preferentemente las unidades del Sistema Internacional. Para cada indicador es necesario seleccionar la relativización más apropiada, de acuerdo con los siguientes criterios generales:

- Los valores relativos por superficie son más apropiados para los indicadores ambientales (erosión, vegetación, fauna,...) pues tienen una fuerte componente territorial.

- Los valores relativos por población se utilizarán principalmente para las variables sociales (parámetros demográficos, renta,...).

- Los valores relativos por superficie y población sirven para indicadores con fuerte implicación territorial y social y de interés relevante.

- Las variaciones temporales son útiles en todos los indicadores pues indican su tendencia, sobre todo en aquellos que demostraron ser especialmente variables en el tiempo.

- Los valores que se expresen en función de otro indicador son especialmente apropiados para los indicadores de respuesta institucional, pues miden su eficacia. Por ejemplo, frente al indicador de presión ambiental «volumen de aguas residuales», expresaremos en porcentaje (\%) el indicador de respuesta en función de la presión, esto es «volumen de aguas residuales que son tratadas».

- Por otra parte, la densidad superficial es más fiable que la densidad por población pues el dato de la superficie lo es en relación con el de población. 
- Asimismo, los indicadores cuyos datos se obtengan por estimación indirecta en función de la población no se expresan en términos relativos poblacionales por carecer de sentido.

Por último, para evitar valores excesivamente altos o bajos que dificulten su manejabilidad, se recomienda ponderarlos, magnificando o reduciendo el valor de forma lineal, esto es, multiplicando o dividiendo por una constante, de forma que los valores se muevan en una franja entre dos o tres decimales y mil unidades (0,01-1000); la homogenización debe ser igual para todos los indicadores similares dentro de un mismo bloque, con el objetivo de facilitar el trabajo de comparación y agregación de indicadores.

\subsection{Aplicación y desarrollo de los indicadores en las áreas geográficas}

Para el cálculo de cada indicador y en cada área de estudio se podrá utilizar el siguiente esquema de trabajo:

- Obtención y exposición de una serie suficiente de datos del área.

- Obtención de valores estadísticos, característicos y singulares.

- Obtención de los valores relativos en todas las áreas unitarias.

- Representación gráfica (p.ej. media móvil) de los Indicadores relativos.

- Regresión de los valores totales del área, y estimación de valores futuros.

- Cálculo de los Indicadores Relativos seleccionados.

- Selección del año o años más adecuados para la comparación sincrónica entre áreas.

Se realzarán los valores más característicos y singulares.

\section{Obtención y estimación de los valores umbrales y objetivos}

En este apartado se intentarán obtener los valores umbrales y objetivos, para un área concreta, o para un conjunto de áreas de características similares, o incluso para áreas geográficas superiores que tendrán carácter de referencia.

\subsection{Valores de Referencia}

Para la obtención de valores umbrales y objetivos, se tendrán en cuenta los Valores de Referencia que permitan realizar una primera estimación o aproxi- 
mación, mediante la evaluación en términos de distancia. Estos valores de referencia pueden ser:

- Valores normativos o propuestos por la literatura científica:

- Valores Legales: o combinación de valores presentes en leyes o tratados regionales, nacionales e internacionales.

- Valores Administrativos, recomendados por organismos especializados, establecidos en Políticas, Planes, Estrategias,...

- Valores establecidos o propuestos en otros modelos de indicadores y en trabajos científicos.

- Valores Medios:

- Espaciales: áreas de la Red, superiores al área (Provincial, Regional, Nacional, Internacional, ...), otras áreas similares, etc.

- Medias temporales: de las áreas de la Red, de otras áreas,...

- Opinión de expertos, representantes institucionales, científicos, actores sociales, opinión popular, etc.

\subsection{Estimación de Valores Umbrales: deseables y críticos}

El valor umbral es aquel que se considera límite desde el punto de vista de la sostenibilidad. Por tanto, en principio, existirá un valor límite negativo, o crítico, y otro valor óptimo, o deseable. A partir de los valores anteriores, se propondrá una jerarquía de valores, obtenida de forma razonada y motivada, en:

- Valor Deseable (u Óptimo)

- Valor Crítico (negativo)

El primero se define como el valor numérico normativo de un indicador de sostenibilidad que asegura el equilibrio con la capacidad de carga del medio ambiente de la región objeto de estudio (Nijkamp y Vreeker, citados por Castro, 2004). Al no existir una medida oficial operativa del desarrollo sostenible, en la mayoría de estudios se elaboran índices basados en una definición relativa, comparando a la mejor situación existente en el ámbito de estudio o superior, para cada indicador de base (Castro, 2004).

El segundo indica el mínimo o máximo valor que puede tener un indicador, bajo o sobre el cual existe una clara situación de insostenibilidad, por tanto de signo contrario a los valores deseables que se corresponden con una situación positiva que se puede alcanzar. 
6.3. Estimación de Valores Objetivo o de aproximación pragmática hacia valores deseables

Los valores objetivos aluden específicamente a la intención, representando un valor (o intervalo), no necesariamente observado, que se espera alcanzar como objetivo final de la política a implementar (Castro, 2004). Se determinan para fechas o periodos determinados, deben ser valores realistas, posibles de alcanzar, y que signifiquen progresos razonables hacia el umbral deseable de sostenibilidad. Se estiman, por tanto, a partir de este último.

Partiendo de los valores de referencia y de los valores deseables obtenidos de forma razonada y de los periodos y valores objetivos de progresos considerados en Instituciones, organismos, otros modelos,... que se extraerán de la literatura científica y de Instituciones mundialmente reconocidas como: ONU, OCDE, AEMA, OSE, Estrategias de gobiernos, etc., se fijará el periodo de referencia o intervalo de tiempo considerado para alcanzar la meta, y se hará una propuesta realista y pragmática de valores objetivos que deberán alcanzar los indicadores en el periodo determinado.

\section{Discusión e interpretación de los resultados}

Los trabajos concretos para el desarrollo de esta tarea se diferenciarán según dos tipos de especializaciones:

1. Especialización Geográfica (EG).

2. Especialización Temática (ET).

En el primer caso, se elaborará una valoración global de la sostenibilidad de cada Área Temática en los espacios rurales, relacionando los resultados de los distintos Indicadores y Conjuntos de Indicadores, y resaltando los elementos más significativos en las áreas piloto de aplicación. Esta valoración se debe hacer en función de los criterios y límites de la sostenibilidad (económica, social, ambiental e integrada) fijados inicialmente y de las consideraciones y conclusiones obtenidos en otros modelos. En particular, se pondrán de manifiesto:

- La evolución en periodos pasados.

- Para el periodo actual y futuro:

- Situaciones claramente insostenibles o aspectos a mejorar.

- Situaciones sostenibles o signos para la esperanza. 
En el segundo caso, para cada una de los áreas geográficas de estudio consideradas se evaluará la sostenibilidad (económica, social, ambiental e integrada o global) del desarrollo en relación con el Indicador de acuerdo con los valores obtenidos, los valores umbrales estimados, y los criterios y límites de la sostenibilidad (económica, social, ambiental e integrada o global) fijados inicialmente.

\section{Indicadores de desarrollo sostenible: un resumen}

La presentación de los indicadores que a continuación se muestra, sigue el esquema propuesto por EUROSTAT en su publicación Measuring progress towards a more sustainable Europe. Proposed indicators for sustainable development. Esta estructura es heredera de las dimensiones que subyacen al concepto de Sostenibilidad y permite identificar cuatro grandes áreas - Social, Económica, Medioambiental e Institucional- en las cuales se ubican los indicadores. A su vez, en cada área los indicadores se clasifican por temas y subtemas. La ubicación de un indicador en un área determinada no es excluyente para que esté relacionado con otras, de forma que podremos encontrar indicadores del ámbito social intrínsecamente ligados con los del ámbito económico. Esto es así por la propia naturaleza del problema que se pretende medir, en el cual en ocasiones todas las dimensiones confluyen sobre un mismo eje. En cuanto al cálculo de indicadores se hace imprescindible seguir una metodología armonizada y única que permita garantizar la comparabilidad de los resultados entre unas áreas y otras. En este caso se han seguido las directrices establecidas por EUROSTAT que han sido aceptadas internacionalmente. La armonización de métodos y nomenclaturas tiene indudables ventajas que han sido ampliamente elogiadas y las cuales compartimos en su totalidad. Sin embargo, la armonización metodológica también presenta limitaciones que es importante tener presente. La principal, hace referencia al uso de fuentes estadísticas que han sido diseñadas para áreas nacionales y cuya utilidad para espacios geográficos más pequeños como es el caso de las regiones conlleva problemas asociados al tamaño de muestras y consecuentemente a la fiabilidad de los resultados.

Por suerte este problema solo afecta a aquellos indicadores que toman su información de encuestas diseñadas para ámbito nacional y no influye en ninguna medida sobre el resto. Al mismo tiempo se incluyen comentarios metodológicos que enriquecen la interpretación de cada indicador. Uno de los ejes de la política europea en materia de Medio Ambiente es el proceso de integra- 
ción de las variables ambientales, conocido como proceso de Cardiff. Por esta razón, en materia de estadística e información ambiental de diversos organismos e instituciones comunitarios se están centrando en el desarrollo de indicadores que permiten analizar y evaluar el proceso de integración a la vez que ofrecer una información sobre el estado y tendencias del medio ambiente de forma simple y sencilla.

El PNB se muestra como un indicador económico incapaz de hacer frente a la representatividad de la economía real, en cuanto a su relación con el sistema ambiental. Por esta razón, la doctrina ha comenzado a elaborar nuevos indicadores más apropiados a una realidad más compleja que la definida por la economía. Los sistemas naturales y los sistemas económicos funcionan como sistemas abiertos, que además están interrelacionados; los indicadores deben reflejar estas relaciones dentro de un marco integral. Podemos encontrar muchos ejemplos de indicadores representativos.

\section{A. Índice de Desarrollo Humano (IDH)}

Este índice apareció en el Informe sobre Desarrollo Humano de 1990 del PNUD. Clasifica el nivel relativo de desarrollo de los distintos países ajustando la renta nacional per capita para explicar las diferencias existentes entre los países en tipos de cambio y poder de compra, utilizando variables como la esperanza de vida, el analfabetismo y la mediana de años de escolarización, junto a la renta per cápita. Es un indicador de desarrollo humano, por lo que no hace referencia expresa al medio ambiente, aunque ha ido evolucionando su contenido y va incorporando diferentes aspectos; así, en el año 1995 se tomó en consideración la condición de la mujer.

\section{B. Índice de bienestar Económico Sostenible (IBES)}

Este índice pretende introducir modificaciones en la valoración del PNB con objeto de contemplar la economía no registrada por el mercado, sector informal, como es el trabajo doméstico. Además incluye las externalidades ambientales, los recursos naturales y los bienes de la biosfera, aunque la valoración de estos fenómenos se hace bastante problemática. 


\section{C. Índice de Sostenibilidad Ambiental (ESI)}

El Ministerio de Medio Ambiente, participando en diversas iniciativas internacionales, se ha planteado la conveniencia de disponer de un documento con información sobre el estado del medio ambiente, basado en la construcción de indicadores, que representen de forma fiel el perfil ambiental de nuestro país y permita fundamentar la toma de decisiones y evaluar la integración del medio ambiente en los sectores de actividad.

Se ha realizado en un proceso de consenso la selección de una serie de indicadores para la Red Europea de Información y Observación del Medio Ambiente (EIONET); y se ha aprobado una primera versión en septiembre de 2000 de los indicadores que se van a utilizar de forma homogénea por todos los Estados de la Unión Europea: son indicadores ambientales, e indicadores socio-económicos y sectoriales (tabla 4).

La necesidad de elaborar un tronco común de indicadores medioambientales se tiene que ir desarrollando de forma paulatina a medida que avanzan diversos sistemas de medición de los aspectos sociales, económicos y ambientales. Recientemente (en enero de 2001) se ha desarrollado una iniciativa en un Foro Económico Mundial en Davos, Suiza, donde se presenta la elaboración de un Índice de Sostenibilidad Ambiental integrado, que va a tener en cuenta diversas dimensiones del medio ambiente (tabla 5).

El Índice de Sostenibilidad Ambiental (ESI) es una medida del progreso hacia un desarrollo sostenible ambiental. Se define Sostenibilidad ambiental como la posibilidad para responder de forma acertada en cada dimensión que afecte al medio ambiente. Un alto ESI indica el camino hacia un alto nivel de Sostenibilidad en términos ambientales, un bajo ESI indicará la existencia de problemas sustanciales de cara a combatir los problemas medioambientales.

El ESI se compone de un conjunto de 22 indicadores, cada uno de los cuales está compuesto de entre dos y seis variables, en total se cuenta con 167 variables. El ESI permite realizar una comparación internacional, y representa un primer paso hacia la búsqueda de herramientas útiles para lograr la toma acertada de decisiones. Este indicador permite:

a) Identificar si los resultados de las políticas ambientales nacionales están por encima o por debajo de sus expectativas.

b) Identificar áreas de éxito y de fracaso de éstas políticas.

c) Comparación en las actuaciones ambientales.

d) Identificar cuáles son «las mejores prácticas disponibles».

e) Investigar acerca de las relaciones existente entre las actuaciones ambientales y las económicas. 
TABLA 4

INDICADORES PARA LA RED EUROPEA DE INFORMACIÓN Y OBSERVACIÓN DEL MEDIO AMBIENTE (EIONET)

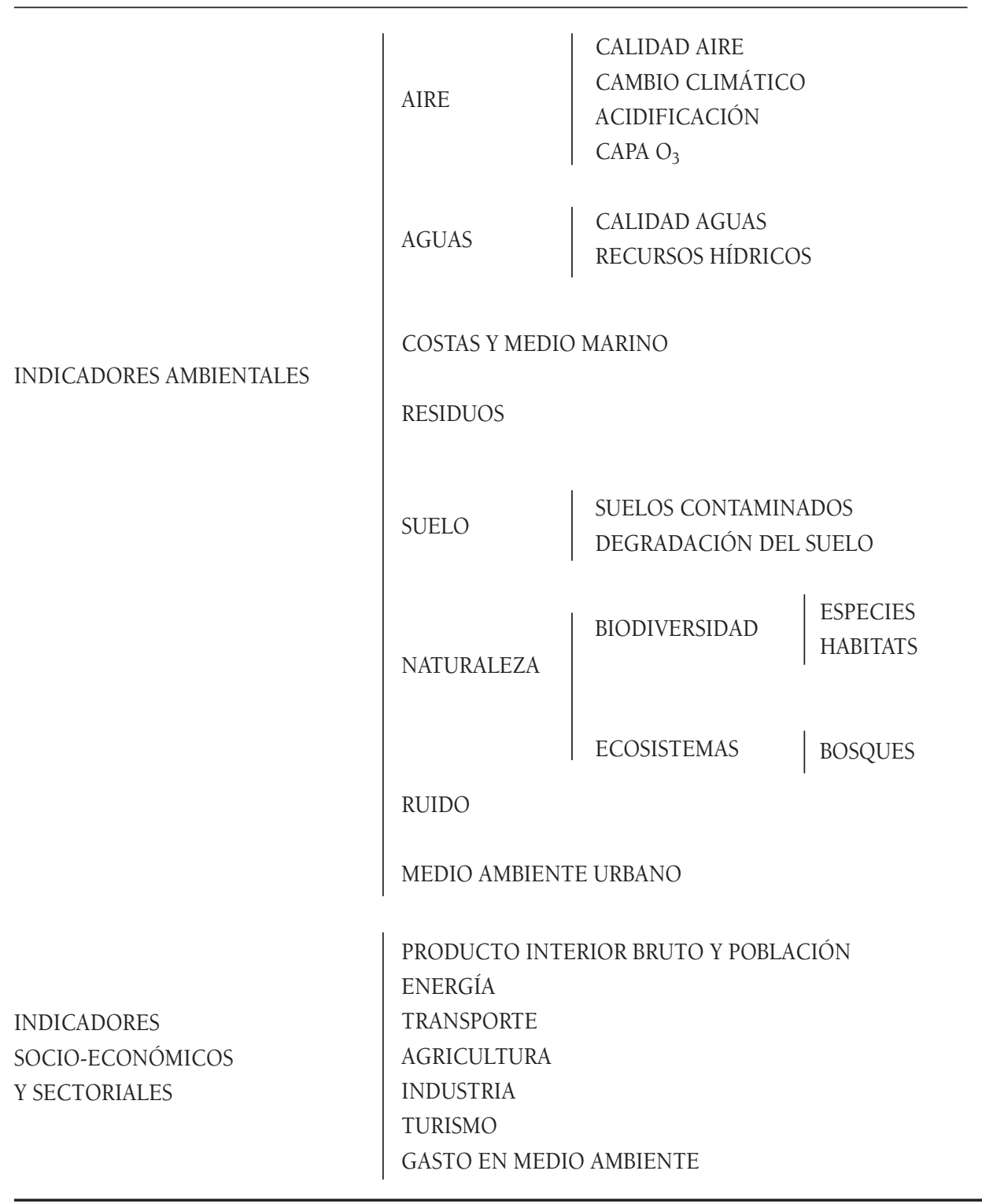

Fuente: Elaboración propia a partir de datos publicados por el Ministerio de Medio Ambiente. 
TABLA 5

DIMENSIONES DEL MEDIO AMBIENTE CONSIDERADAS POR EL ÍNDICE DE SOSTENIBILIDAD AMBIENTAL

Sistema ambiental

Reducción del estrés ambiental

Reducción de la vulnerabilidad humana

Capacidad social e institucional
Un país es ambientalmente sostenible para alcanzar un sistema ambiental vital si mantiene unos niveles de salud, y para alcanzar dichos niveles deberá mejorar más que deteriorarse.

Un país es ambientalmente sostenible si los niveles de estrés antropogénico son suficientemente bajos como para no generar peligro demostrable hacia el medio ambiente

Un país es ambientalmente sostenible si alcanza un sistema social no vulnerable (en el sentido de necesidades básicas tales como nutrición y salud); el ser menos vulnerable es un signo de que la sociedad va por el camino de alcanzar la Sostenibilidad.

Un país es ambientalmente sostenible si tiene instituciones capaces, mecanismos y actitudes que supongan una herramienta efectiva ante cambios en el medio ambiente.

Fuente: Main Report of World Economic Forum, 2004. Environmental Sustainability Index, 2004.

La Sostenibilidad ambiental podrá ser representada como función de cinco fenómenos:

1) El estado de los sistemas ambientales: aire, agua, suelo, ecosistemas.

2) Nivel de estrés de los sistemas en términos de contaminación y niveles de explotación.

3) Vulnerabilidad humana hacia cambios en el medio ambiente.

4) Capacidad social e institucional para adaptarse a cambios en el medio ambiente.

5) Posibilidad de responder a las demandas de un sistema global a través de esfuerzos colectivos (p.e. frente al cambio climático, deforestación...) 


\section{UN ESTUDIO DE CASO: APLICACIÓN DE LA METODOLOGÍA EN ÁREAS RURALES ANDALUZAS DE METODOLOGÍA LEADER}

El proyecto de investigación «Construcción de Indicadores de Sostenibilidad en áreas rurales españolas Leader y Proder - Indi-Rural», realizado por la Universidad de Almería, determinó como ámbito geográfico de actuación ocho áreas rurales Leader y Proder de España, de las cuales, cuatro son comarcas andaluzas: Alpujarra almeriense, Almanzora, Serranía de Ronda y Bajo Guadalquivir. En este estudio se han registrado avances hasta la etapa 3 del modelo propuesto, llegando a construir un primer sistema de indicadores compuesto de 228 indicadores simples, en ocho áreas temáticas, para áreas rurales extensivas y de montaña. En la actualidad se están construyendo los programas informáticos asociados y se está trabajando en la organización y animación del capital social.

En la caracterización del capital social, se ha identificado en España, y concretamente en las comarcas de metodología Leader, como principal Unidad neo-endógena a las asociaciones que gestionan los fondos del FEADER, que normalmente adoptan la personalidad jurídica de Sociedad Anónima con participación mixta: Pública (ayuntamientos, comunidad autónoma, diputación, etc...) y Privada (empresas, asociaciones de productores,...), y que se encargan de promover y cofinanciar Proyectos de desarrollo rural.

En el diagnóstico inicial de las comarcas, se identificó que existen diferencias considerables entre los términos municipales que conforman las comarcas, ya sea por sus características geográficas, políticas, productivas y/o influencias externas. Por ello fue necesario realizar un análisis conjunto de la comarca completa y de la misma sin los municipios que difieren del resto (tabla 6).

Para obtener áreas homogéneas se desarrollaron indicadores caracterizadores como: densidad poblacional, superficie agrícola, número de industrias, número de centros de salud, de centros educativos, entre otros. En base a los resultados obtenidos, se identificaron los términos municipales que difieren del resto, y se procedió a la definición de áreas «completas» y áreas «reducidas», entendiéndose por la primera las áreas rurales compuestas por todos los términos municipales de la comarca, y por la segunda una «subcomarca» conformada por los términos municipales más homogéneos (excluyendo los municipios menos rurales y de montaña).

En el caso de las Alpujarras Almerienses, Berja se destaca por la importancia que ha tomado en los últimos años la agricultura intensiva bajo plástico o en invernadero, se estima que existen cerca de 1.400 ha bajo este sistema de producción, que constituyen aproximadamente el 30\% de la Superficie Agra- 
TABLA 6

SISTEMA DE INDICADORES DE SOSTENIBILIDAD INDIRURAL POR ÁREAS TEMÁTICAS

\begin{tabular}{|c|c|}
\hline Marco de Referencia & Demografía \\
\hline $\begin{array}{l}\text {-Altitud } \\
\text {-Superficie } \\
\text {-Superficie de pastizales } \\
\text {-Superficie de prados naturales } \\
\text {-Superficie agraria utilizada (SAU) } \\
\text {-Superficie de tierras labradas } \\
\text {-Superficie para pastos permanentes } \\
\text {-Superficie otras tierras forestales } \\
\text {-Superficie otras tierras no forestales } \\
\text {-Número de viviendas totales }\end{array}$ & $\begin{array}{l}\text {-Población, por sexo y por edad } \\
\text {-Tasa bruta de natalidad } \\
\text {-Tasa bruta de mortalidad } \\
\text {-Tasa de mortalidad infantil } \\
\text {-Crecimiento vegetativo } \\
\text {-Tasa de envejecimiento } \\
\text {-Emigración interior total } \\
\text {-Inmigración interior total } \\
\text {-Inmigrantes extranjeros total } \\
\text {-Saldo migratorio }\end{array}$ \\
\hline Medio Laboral & Economía Local \\
\hline $\begin{array}{l}\text {-Población activa } \\
\text {-Población activa ocupada } \\
\text {-Población activa parada } \\
\text {-Paro total registrado } \\
\text {-Paro total registrado femenino } \\
\text {-Renta familiar disponible por habitante } \\
\text {-IRPF: \# de declaraciones } \\
\text {-IRPF: Rentas netas declaradas } \\
\text {-U. de trabajo en explotaciones agrarias } \\
\text {-Sociedades laborales totales } \\
\text {-Número de cooperativas totales } \\
\text {-Número de cooperativas agrarias }\end{array}$ & $\begin{array}{l}\text {-I.B.I. Urbano - No de recibos } \\
\text {-I.B.I. Rústico - No de titulares } \\
\text {-Ingresos fiscales por habitante } \\
\text {-Presupuesto liquidado de ingresos } \\
\text {-Presupuesto liquidado de gastos } \\
\text {-Déficit o Superávit del presupuesto } \\
\text {-Locales activos total } \\
\text {-Situaciones de altas en el IAE } \\
\text {-Inversiones agrícolas } \\
\text {-Inversiones en construcción } \\
\text {-Inversiones en industria } \\
\text {-Inversiones en servicios }\end{array}$ \\
\hline Turismo, Energía y Transporte & Agricultura y Ganadería \\
\hline $\begin{array}{l}\text {-Capacidad de Alojamiento total } \\
\text {-Parque de Vehículos totales } \\
\text {-Red viaria total } \\
\text {-Número de núcleos Urbanos } \\
\text {-Vertebración territorial } \\
\text {-Consumo de energía eléctrica } \\
\text {-Consumo de energía eléctrica } \\
\text { residencial } \\
\text {-Consumo de energía eléctrica } \\
\text { agrícola }\end{array}$ & $\begin{array}{l}\text {-Explotaciones agricultura ecológica } \\
\text {-Censo de bovino } \\
\text {-Censo de ovino } \\
\text {-Censo de caprino } \\
\text {-Censo de porcino } \\
\text {-Censo de aves } \\
\text {-Censo de equinos } \\
\text {-Censo de conejas madres }\end{array}$ \\
\hline
\end{tabular}




\begin{tabular}{ll}
\hline \multicolumn{1}{c}{ Medio Natural } & \multicolumn{1}{c}{ Medio Social } \\
\hline -Índice de erosión ponderado & -Centros públicos de educaciónbásica \\
-Suelos según pendiente & -Alumnos por centros públicos de \\
-Emisiones de CO & educación primaria \\
-Volumen de agua abastecido al total & -Centros públicos secundarios \\
de la población & -Alumnos por centros públicos de \\
-Volumen de agua facturada por & educación secundaria \\
municipio & -Centros de educación de adultos \\
-Producción anual de residuos sólidos & -Alumnos por centros públicos de \\
-Eliminación de RSU en vertederos & educación de adultos \\
controlados & -Recursos de atención primaria totales \\
-Superficie forestal & -Recursos de atención primaria - centros \\
-Número de especies de avifauna & de salud \\
amenazada & -Centros asistenciales total y desglosado \\
-Superficie total Espacios naturales & -Índice de participación en elecciones \\
Protegidos (ENP) incluidos en el & locales \\
listado de lugares de interés & \\
comunitario (LICs) & \\
\hline
\end{tabular}

ria Utilizada (SAU) del municipio, y el 15\% de la comarca. Macael y Olula del Río, en la comarca del Almanzora, basan su economía en las canteras de mármol y en sus actividades auxiliares. Esta actividad económica les ha permitido destacarse dentro de la comarca y convertirse en su polo de desarrollo, hecho que refleja el análisis del indicador altas en el Impuesto de Actividades Económicas en la sección de extracción y transformación de minerales no energéticos y productos derivados, cuyos resultados arrojan que ambos municipios concentran el 61,12\% (2005) de las altas de la comarca.

La SAU en el término municipal de Ronda (Serranía de Ronda) abarca el $80 \%$ de su superficie, que representa al 30\% de la comarca, dato que corrobora el fuerte componente agrícola del municipio, y actividad en la que basa su economía. También se destaca el turismo rural como principal medio de diversificación de la economía. La ubicación geográfica de la comarca del Bajo Guadalquivir, en la margen izquierda del río del mismo nombre, favorece el desarrollo de las actividades agrícolas. Utrera es el núcleo de mayor concentración socioeconómica, principalmente por sus tierras fértiles y la buena comunicación por carretera y ferrocarril existente ( $16 \%$ de la SAU de la comarca, y 78,47\% del municipio). Cabe destacar también el alto dinamismo económico que posee San Lúcar de Barrameda, debido a las actividades agrí- 
colas propias de la comarca y por el crecimiento del sector industrial emergente, diversificando la estructura productiva y mejorando el nivel de vida de sus habitantes.

En el análisis de los resultados preliminares obtenidos en el cálculo de los indicadores, observamos que en la dimensión socio-económica se evidencia el envejecimiento de la población en las áreas rurales, con porcentajes superiores a la media española 16,90\% (INE, 2009) en el caso de las comarcas de la Alpujarra almeriense, Almanzora y Serranía de Ronda, tanto las áreas completa como reducida (figura 4). La comarca del Bajo Guadalquivir presenta porcentajes inferiores debido a una mayor tasa de natalidad y un aumento de la población comprendida entre los 0 y 14 años. En general la población de estas áreas rurales está comprendida entre los 20 y 64 años (más del 60\%), que indica un porcentaje de población activa superior al resto de comarcas (figura 5). Pero hay que considerar que alrededor del $40 \%$ está comprendida entre los 35 y 64 años, que a futuro constituye un mayor envejecimiento de la población, con los inconvenientes que esto representa en el desarrollo de las activi-

FIGURA 8

\section{DISTRIBUCIÓN PORCENTUAL DE LA POBLACIÓN DE LAS CUATRO COMARCAS ANDALUZAS (AÑO 2004)}

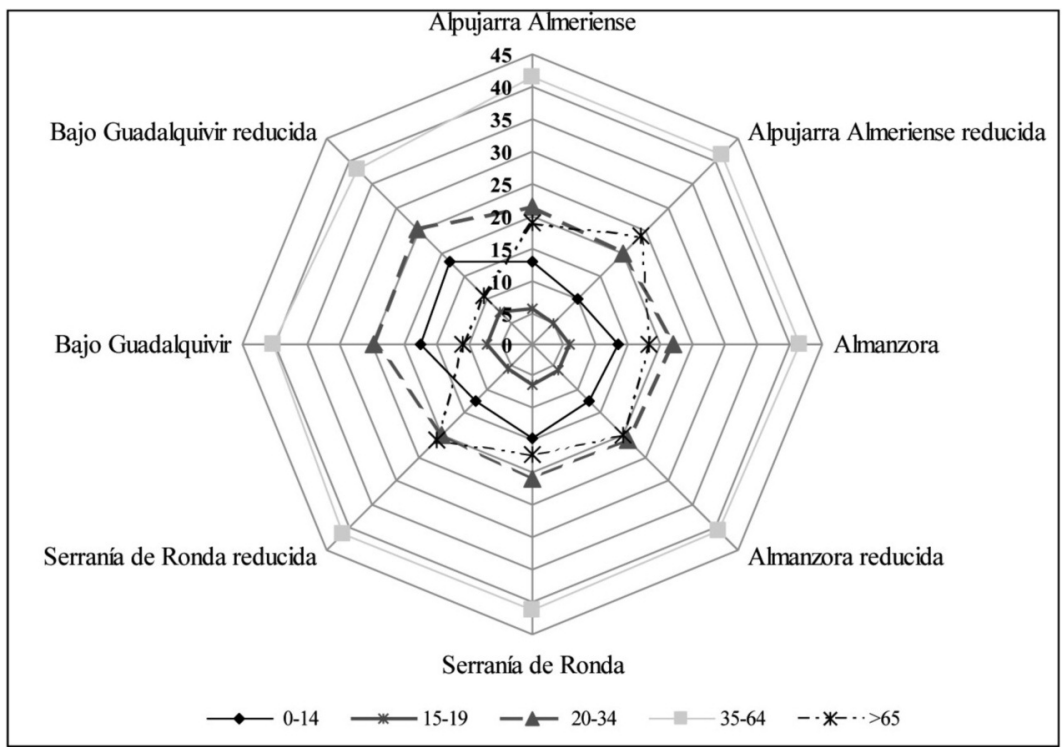

Fuente: Elaboración propia. 
dades productivas. En todas las comarcas se observa que la población activa masculina es superior con el doble, e incluso el triple, en el caso de la comarca del Bajo Guadalquivir, el porcentaje de población activa femenina. El paro total registrado es inferior en las comarcas almerienses (Alpujarra y Almanzora) a la media española y andaluza (10,69 y $11,64 \%$ de la población activa respectivamente), y similar en las otras dos comarcas.

En la dimensión ambiental, se observan grandes diferencias entre las comarcas de interior y la comarca del Bajo Guadalquivir, donde solo el 5,37\% de la SAU es de tipo forestal, debido principalmente al fuerte componente agrícola de la comarca. En todas las comarcas, predominan las formaciones arbustivas y herbáceas sin arbolado, seguido de las formaciones de matorral con arbolado. En las comarcas almerienses existe un porcentaje considerable de espacios abiertos con escasa cobertura vegetal, debido a las condiciones climáticas y edafológicas de la zona.

\section{FIGURA 9}

\section{DISTRIBUCIÓN PORCENTUAL DE LA POBLACIÓN ACTIVA DE LAS CUATRO COMARCAS ANDALUZAS (AÑO 2001)}

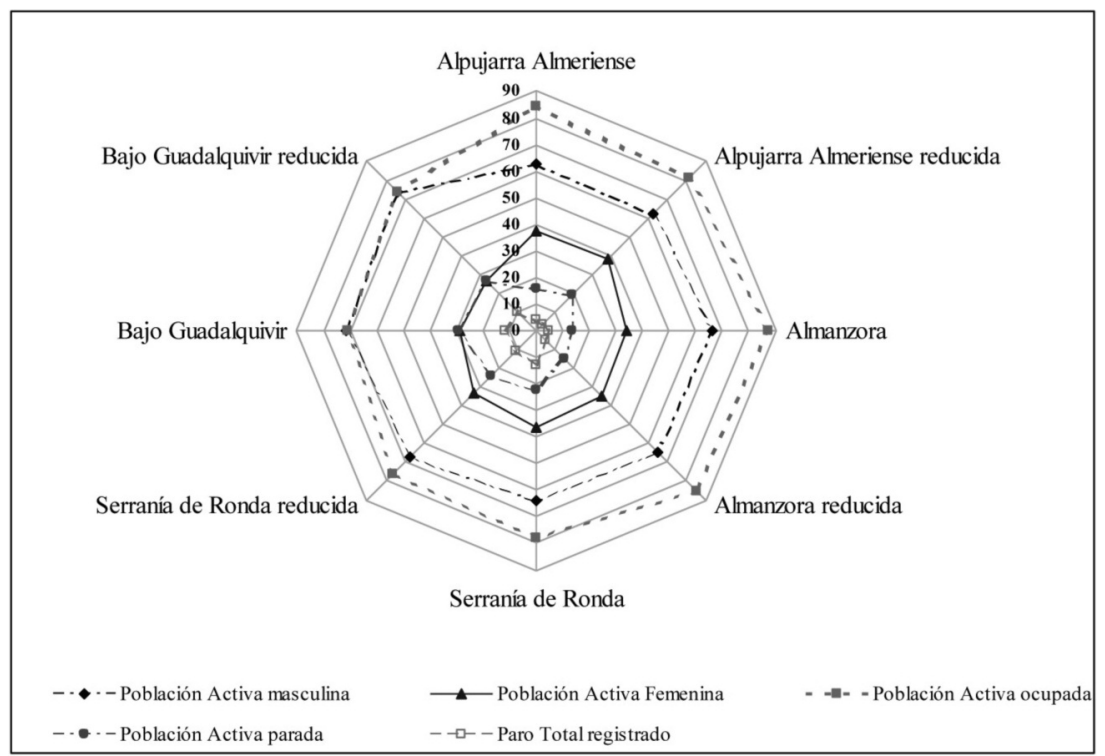

Fuente: Elaboración propia. 
FIGURA 10

SUPERFICIE FORESTAL Y DISTRIBUCIÓN PORCENTUAL DE LA DISTINTAS FORMACIONES FORESTALES DE LAS CUATRO COMARCAS ANDALUZAS (AÑO 2004)

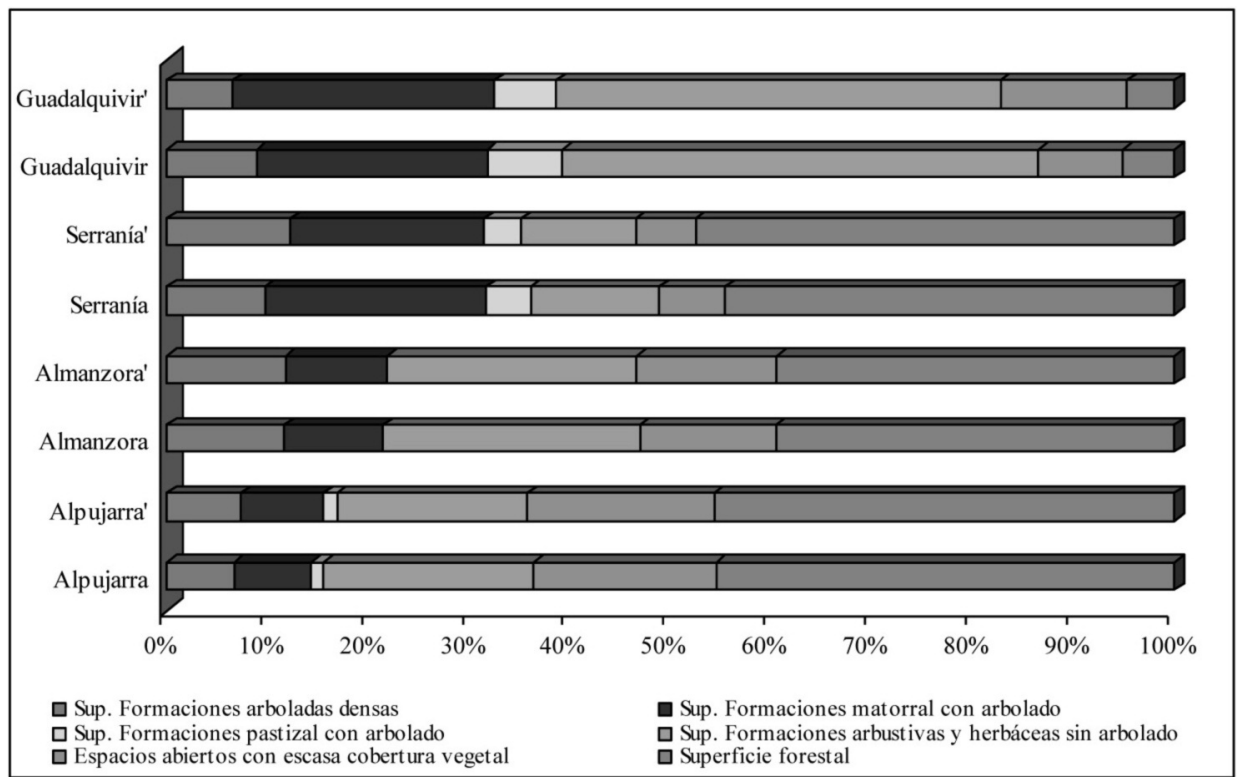

Fuente: Elaboración propia.

\section{A MODO DE CONCLUSIONES}

La mundialización y globalización del desarrollo sostenible requiere la utilización de Indicadores transferibles que permitan a los actores involucrados situar sus estrategias con respecto a otros lugares. Sin embargo, la mayor parte de los Sistemas de Indicadores han sido construidos mediante aproximaciones top-down, lo que les resta legitimidad con respecto a las partes interesadas.

Las metodologías propuestas se insertan en una corriente importante e innovadora sobre modelos de indicadores (OCDE, ONU, AEMA, MMA, OSE,...) en constante proceso de actualización y renovación. El interés creciente en la definición de sistemas de indicadores revela su importancia en la incorporación de los principios de la sostenibilidad a las distintas políticas horizontales y sectoriales. Sin embargo, todavía existen lagunas en la definición y aplica- 
ción de Indicadores de Sostenibilidad de carácter local, y muy especialmente en áreas rurales.

Se han sentado las bases metodológicas comunes a cualquier sistema de Indicadores de Sostenibilidad, especificando, en particular, los principios y las características, que deberían cumplir todos ellos, sobre:

- La generación y la clasificación de indicadores, que permita la especialización por áreas temáticas.

- La selección de indicadores, a través una caracterización y posterior valoración mediante atributos.

- La construcción de los indicadores seleccionados, en particular, la construcción técnica de los indicadores relativos.

- La aplicación y el desarrollo del indicador, y la presentación de los resultados.

- La aproximación a los valores umbral deseable y a los valores objetivos de progresos realistas hacia la sostenibilidad.

La homogeneidad y uniformidad en la construcción de sistemas de indicadores de sostenibilidad contribuirá a que pueda haber entre ellos una Integración Vertical, esto es, entre sistemas de escala superior (continentales, nacionales,...) e inferior (municipales, comarcales, ...), y una Integración Horizontal, es decir, entre sistemas sectoriales: de turismo, agrícola,...

El desarrollo parcial de la metodología nos ha permitido detectar nuevas necesidades y orientaciones para futuras investigaciones, entre las que destacamos:

- Homogeneizar y unificar el lenguaje y la terminología utilizada en los Sistemas de Indicadores (hoy muy dispersa y heterogénea).

- Mejorar la arquitectura informática de difusión de la información, con una estructura más homogénea, y desarrollar tecnologías innovadoras de información como por ejemplo WEB con estructura semántica.

- Profundizar en el carácter local de los Sistemas de Indicadores, en particular para municipios rurales. Para ello, es necesario reducir el número de Áreas Geográficas y abordarlas de forma más intensa.

- Incrementar el número de datos de carácter local (p. ej. datos de percepción,...) lo que requiere un mayor trabajo de campo.

- Diseñar nuevos Indicadores:

- A partir de los nuevos datos.

- Por agregación de Indicadores ya definidos (obteniendo «cestas» de indicadores).

- Por nueva selección (pues la realidad es cambiante). 
- Aplicar y desarrollar los Indicadores en nuevas áreas rurales de naturaleza distinta, como son las áreas de agricultura intensiva, para contrastar estos nuevos sistemas de indicadores con los establecidos en áreas rurales extensivas y de montaña (Leader).

- Incrementar la participación de la población y de los actores sociales claves en la construcción de los Indicadores (en todas las etapas del proceso: generación, caracterización, selección, aplicación y uso).

- Implicar a las Instituciones Públicas y Privadas locales en el Uso de los Sistemas de Indicadores, sometiéndolos a debates y dotándolos de legitimidad en la toma de sus decisiones.

El desarrollo futuro de los sistemas locales de indicadores permitirá implicar a las Instituciones Públicas y Privadas locales en su uso, para el seguimiento y control de sus instrumentos legales y administrativos (Estrategias, Planes Sectoriales: Turismo, Agricultura, Transporte, Energía,... Planes de Ordenación Territorial de las Comarcas rurales, Red de Municipios Sostenibles,...).

Recibido: 15/06/2009

Aceptado: 30/06/2010

\section{BiBLIOGRAFÍA GENERAL}

AEMA (Agencia Europea de Medio Ambiente, Unión Europea) (2004): Estrategia de la AEMA 2004-2008. Copenhague, Agencia Europea de Medio Ambiente.

AEMA (2005): El medio ambiente europeo, estado y perspectivas 2005. Copenhague, Agencia Europea de Medio Ambiente.

AEMA (2007): Consultado: Septiembre 20, 2007. Disponible en: http://www.eea.eu ropa.eu. Dinamarca.

AEMA (2009): Señales de la AEMA 2009, cuestiones medioambientales de capital importancia para Europa. Copenhague, Agencia Europea de Medio Ambiente. Disponible en http://www.eea.europa.eu/es/publications/signals-2009 EEA-Signals-2009ES-web.pdf (consulta 25/08/2011).

AEMA (2009): EU Biodiversity Action Plan. Copenhague, Agencia Europea de Medio Ambiente. Disponible en http://ec.europa.eu/environment/nature/biodiversity/ comm2006/index_en.htm (consulta 25/08/2011).

Bakkes, J. et al. (1994): An overview of environmental indicators: state of the art and perspectives. Bilthoven, National Institute of Public Health and Environmental Protection. Disponible en http://www.rivm.nl/bibliotheek/rapporten/402001001.html (consulta 25/08/2011). 
Bossel, H. (1999): Indicators for Sustainable Development. Theory, Method, Applications. Winnipeg (Manitoba, Canada), Internacional Institute for Sustainable Development. Disponible en http://www.iisd.org/pdf/balatonreport.pdf (consulta 25/08/2011).

Castro, M. (2004): Indicadores de Desarrollo Sostenible Urbano, Una aplicación para Andalucía. Instituto de Estadística de Andalucía. Disponible en www.eumed.net/tesis/jmc/ (consulta 25/08/2011).

CSD (2006a): Global Trends and Status of Indicators of Sustainable Developments. Nueva York, UN Department of Economic and Social Affairs. Disponible en http://www.un.org/esa/sustdev/csd/csd14/documents/bp2_2006.pdf (consulta 25/08/2011).

CSD (2006b): «Expert Group Meeting on Indicators of Sustainable Development. Report». Nueva York, UN Department of Economic and Social Affairs.

CSD (2007): CSD indicators of sustainable development. $3^{\text {rd }}$ edition - Fact Sheet. Disponible en http://www.un.org/esa/sustdev/natlinfo/indicators/factsheet.pdf (consulta 25/08/2011).

Eurostat (Comisión Europea) (2007): Sustainable development indicators. Disponible en: http://epp.eurostat.ec.europa.eu (consultado 01/2008).

Fernández, F. (2006): Indicadores de sostenibilidad y medio ambiente; métodos y escala. Sevilla, Consejería de Medio Ambiente - Junta de Andalucía. Disponible en http://www.juntadeandalucia.es/medioambiente/site/web/menuitem.a5664a214f73 c3df81 d8899661525ea0/?vgnextoid=957528c276 cbf010 VgnVCM1000000624e 50 aRCRD (consulta 25/08/2011).

Gallopín, G. (1997): «Indicators and Their Use: Information for Decision-making. Part One-Introduction», en B. Moldan y S. Bilharz (eds.): Sustainability Indicators. A Report on the Project on Indicators of Sustainable Development. Chichester, Wiley, SCOPE 58, pp. 13-27.

Gallopín, G. (2006): «Los Indicadores de Desarrollo Sostenible: Aspectos Conceptuales y Metodológicos», ponencia realizada para el Seminario de Expertos sobre Indicadores de Sostenibilidad en la Formulación y Seguimiento de Políticas. Chile.

Matías, G. (2004): "Los indicadores del Desarrollo Sostenible", ponencia presentada en la VI Reunión de Economía Mundial. Badajoz.

MMA (Ministerio de Medio Ambiente, Gobierno de España) (2000): Indicadores ambientales. Una propuesta para España. Madrid, Ministerio de Medio Ambiente.

MMA (2006): Conjunto básico de indicadores de la AEMA. Madrid, Ministerio de Medio Ambiente.

Naciones Unidas (2001): Indicators of sustainable development: Guidelines and methodologies. Report. Nueva York.

OSE (Observatorio de la Sostenibilidad en España) (2005): Sostenibilidad en España 2005. Madrid, Ministerio de Medio Ambiente-Fundación Biodiversidad-Fundación Universidad de Alcalá-Mundi-Prensa Libros.

OSE (2006): Sostenibilidad en España 2006. Madrid, Ministerio de Medio AmbienteFundación Biodiversidad-Fundación Universidad de Alcalá-Mundi-Prensa Libros. 
OECD (Organisation for Economic Co-Operation and Development) (2003): OECD Environmental Indcators - Development, measurement and use. Reference Paper.

Pearce, D. y Atkinson, G. (1998): The concept of sustainable development: An evaluation of its usefulness ten years after Brundtland. Centre for Social and Economic Research on the Global Environment, University College London and University of East Anglia. Working Paper PA 98-02, Disponible en http://www.sjes.ch/papers/1998-III-2.pdf (consulta 29/08/2011).

Quiroga, R. (2001): Indicadores de sostenibilidad ambiental y de desarrollo sostenible: estado del arte y perspectivas. Santiago de Chile, CEPAL-ECLAC, Serie Manuales, 16.

Reed, M.; Frases, E. y Dougill, A. (2006): "An adaptative learning process for developing and applying sustainability indicators with local communities". Ecological Economics, 59, pp. 406-418.

\title{
RESUMEN
}

Cada vez más necesitamos maneras de asegurar patrones de desarrollo que sean sustentables, es decir, que sean ambiental, social y económicamente apropiados para nosotros y para las generaciones venideras. Los indicadores de desarrollo sustentable son una herramienta que permitiría "aterrizar" al concepto de sustentabilidad, apoyando la manera en que se toman las decisiones. En España existen pocas experiencias sobre el tema. Este artículo presenta el trabajo realizado para desarrollar indicadores de desarrollo sustentable. Se centra en las lecciones que han surgido de la experiencia, discutiendo su integración a la teoría general del desarrollo económico a partir de la constatación de la centralidad que estos temas han adquirido en los enfoques contemporáneos del desarrollo territorial.

PAlabras Clave: indicadores; desarrollo sustentable; España; desarrollo económico regional.

\begin{abstract}
We are increasingly needing ways to secure patterns of development that be sustainable, that is, environmentally, socially and economically appropriate for us and for future generations. Sustainability indicators are a promising tool that would allow us to "land" the concept, supporting the way in which decisions are made. In Spain there are few experiences on the subject. This paper presents the work carried out to develop sustainability indicators. The paper focuses on the lessons that have been drawn from the experience, discussing its possible integration to general theory of economic development, considering the increasing importance that these topics have gained in contemporary approaches of terriorial development.
\end{abstract}

KEY WORDS: indicators; sustainable development; Spain; regional economic development. 